\title{
Study on Driving Stability of Tank Trucks Based on Equivalent Trammel Pendulum for Liquid Sloshing
}

\author{
Xian-sheng Li, Xue-lian Zheng, Yuan-yuan Ren, Yu-ning Wang, and Zhu-qing Cheng \\ College of Traffic, Jilin University, No. 5988 Renmin Street, Changchun 130022, China \\ Correspondence should be addressed to Yuan-yuan Ren; maggie170101@gmail.com
}

Received 17 July 2013; Revised 27 September 2013; Accepted 27 September 2013

Academic Editor: Wuhong Wang

Copyright ( 2013 Xian-sheng Li et al. This is an open access article distributed under the Creative Commons Attribution License, which permits unrestricted use, distribution, and reproduction in any medium, provided the original work is properly cited.

\begin{abstract}
To investigate the driving stability of tank trucks, an equivalent trammel pendulum was utilized to approximately demonstrate the dynamic characteristics of liquid sloshing in a partially filled tank. The oscillation movement of the trammel pendulum in the tank was described under the tank-fixed coordinate system and its motion equation under the noninertia coordinate system was derived using a Lagrangian function. The motion of the pendulum that expresses the fluid cargo dynamic behavior and that of the solid truck was coupled with each other by the tank. Therefore, a tank truck dynamic model was established using Newton's first law and the angular momentum. A typical tank truck was selected and used to study its driving stability under steering angle step test. The study on tankers driving stability is of great importance for evaluating tankers driving safety, investing the main impact factor aspecting tankers driving stability, and developing active/passive roll control systems for them.
\end{abstract}

\section{Introduction}

About $80 \%$ of global chemical and petroleum products are delivered by road tank vehicles. The transportation freight has already reached 4 billion tons per year. In America, tankers make up more than $55 \%$ of all freight trucks. Tankers are hugely convenient for fluid material exchange and have a positive effect on boosting the national economic development. However, they also create severe traffic safety problems which would result in huge people injury and property damage. Statistical data collected by Statistique Canada has shown that $83 \%$ of lorry rollover accidents on highways are caused by tank vehicles [1]. Meanwhile, in 2011, 416 tanker accidents occurred in China, resulting in more than 400 people being injured or killed as well as immense economic losses. Besides this, for the particularity of liquid cargoes, the release of fluid cargo in tanker accidents could cause contamination to the roads, the water, and the air [2]. Therefore, great attention must be paid to tanker driving safety.

Much works have been carried out on the characteristics of tanker accidents, in an attempt to investigate the primary accident type. It was found by Treichel et al. that rollover is the most frequent accident type for tankers [3], comprising $45.16 \%$ of all tanker accidents in China in 2010. Furthermore, nearly $61 \%$ of tanker rollovers occurred on curved sections of highway. Many researchers have studied the factors behind this phenomenon and have concluded that liquid sloshing in a partially filled tank is the main cause [4-7]. Due to the difference in liquid cargo densities and limitations on vehicle axle loads, tanks are in a partially filled condition most of the time. Due to the existence of liquid-free sloshing space, transient liquid sloshing is produced when vehicle driving state changes $[8,9]$. The lateral sloshing force that acts on the tank wall increases vehicle rollover torque and degrades vehicle roll stability.

To explore the influence of transient liquid sloshing on tanker roll stability, many research works have been explored and some important conclusions have been drawn. Considering the complexity of tank vehicle dynamic analysis-as tank vehicles are fluid-solid coupling multibody systemsome important simplifications have to be made before the analysis. Strandberg et al. assumed that liquid free surface is a tilted straight line whose gradient is a function of vehicle's lateral acceleration and tank's roll angle [10-13]. Based on that, the center of gravity (CG) of liquid bulk can be obtained, and a quasi-static (QS) dynamic analysis of vehicle driving stability can be carried out. The QS method was popular in the initial research phase of the late 1990s, 
and many improved algorithms have been produced to increase the analysis precision to some degree [14, 15].

To investigate the accuracy of the QS method, some researchers studied the relationship between the evaluated liquid sloshing effect obtained by calculating the movement of the CG of the liquid bulk and the observed effect. They have found that the mean values of sloshing forces and the coordinates of the CG of the liquid bulk are quite close between the estimated and observed cases [16]. This result demonstrates the correctness of the QS method, which mean that the worst vehicle driving state can be predicted by the QS method [17].

However, the QS method cannot reflect the dynamic characteristics of liquid sloshing. Therefore, an equivalent mechanical model which can precisely demonstrate the dynamic characteristic of liquid sloshing in a partially filled tank must be modeled first [18-21]. In estimating the tanker's dynamic analysis using equivalent mechanical model for liquid sloshing, the biggest difficulty for researchers is the coupling of the fluid cargo and the solid vehicle. To solve this problem, some people analyzed tanker driving stability in multibody analysis software, and some used the contractive analysis method [20,22]. Neither method had the advantages of the equivalent mechanical model for liquid sloshing, as a vehicle's driving stability cannot be precisely described by the analytical model for vehicle roll stability control system.

Therefore, the purpose of this paper is to establish a tank vehicle dynamic model based on the equivalent mechanical model for transient liquid sloshing in a partially filled tank. The trammel pendulum was used to describe liquid sloshing, its motion was analyzed, and the motion equation was derived under the tank-fixed coordinate. Then, the coupling condition for the fluid cargo and the solid vehicle was studied, and a tanker dynamic model was established based on this. Finally, MATLAB was used to simulate the roll stability of a typical tank truck under steering angle step test, and some important conclusions were drawn. The research achievement is of great significance for commanding and evaluating tank trucks roll stability performance, judging the conditions under which a tanker will lose its driving stability and designing roll stability control systems for tankers to improve their driving safety.

\section{Equivalent Trammel Pendulum for Liquid Sloshing in a Partially Filled Tank}

2.1. The Basic Motion Equation and Parameter Values of the Trammel Pendulum. Theoretical analysis and experimental studies had revealed that the first-order sloshing mode, which can be described by the oscillation of liquid-free surface, is the most important mode of liquid sloshing in partially filled tanks $[20,21]$. MATLAB's numerical method was used to calculate the trajectory of the CG of the liquid bulk that oscillates in a tank with an elliptical or circular cross section. The results showed that the trajectory of the CG of the liquid bulk remains parallel to the tank's periphery [21], which is presented in Figure 1. According to this fact, the trammel pendulum model whose swing trajectory is elliptic

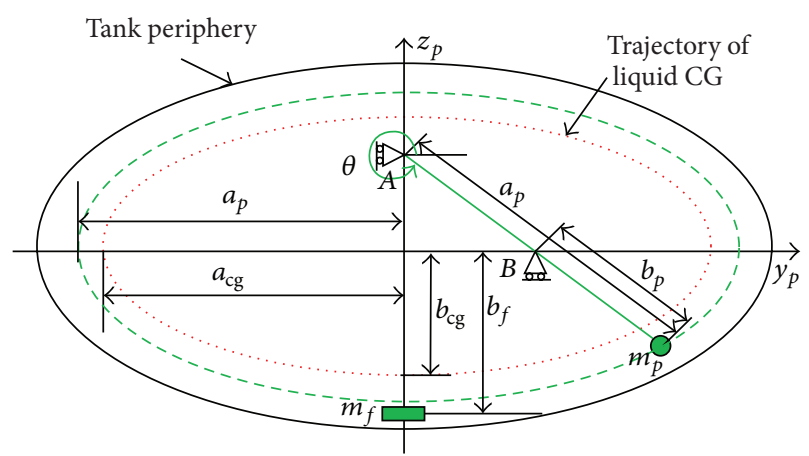

FIgURE 1: Schematic diagram for the motion of the trammel pendulum.

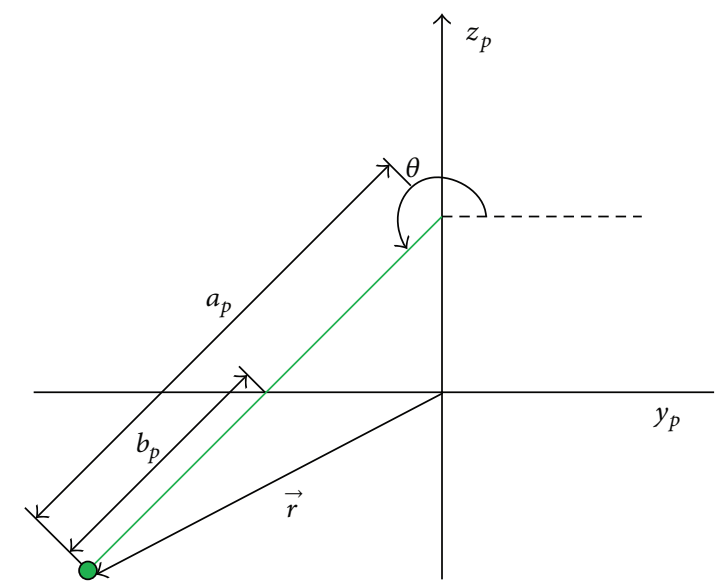

FIGURE 2: Analytical diagram for the motion of the trammel pendulum under an inertial coordinate system.

is established to approximately simulate liquid sloshing in a partially filled tank.

In Figure $1, a_{p}$ is half of the major axis of the pendulum's oscillation trajectory and $b_{p}$ is half of its minor axis. $a_{\mathrm{cg}}$ is half of the major axis of the elliptical trajectory of the CG of the liquid bulk and $b_{c g}$ is half of its minor axis. $m_{p}$ is the pendulum mass, which is equal to the sloshing liquid mass; $m_{f}$ is the fixed liquid mass, which does not participate in sloshing. $\theta$ is the pendulum's amplitude.

The Lagrangian function was used to derive the kinetic equation of the trammel pendulum system. The motion equation of the trammel pendulum that oscillates under tank fixed inertia coordinate system whose origin locates at the center of the tank (as shown in Figure 2) is written as follows:

$$
\begin{aligned}
& \ddot{\theta}\left(a_{p}^{2} \sin ^{2} \theta+b_{p}^{2} \cos ^{2} \theta\right)+\frac{1}{2} \dot{\theta}^{2}\left(a_{p}^{2}-b_{p}^{2}\right) \sin 2 \theta \\
& \quad+g b_{p} \cos \theta=0 .
\end{aligned}
$$

The pendulum parameters in (1) are presented as follows:

$$
\begin{aligned}
\frac{b_{p}}{b}= & 1.089+0.726 \Delta-0.1379 \Lambda-0.953 \Delta^{2}-1.216 \Lambda \Delta \\
& +0.05141 \Lambda^{2}-0.06107 \Delta^{3}+0.5739 \Lambda \Delta^{2}+0.1632 \Lambda^{2} \Delta,
\end{aligned}
$$




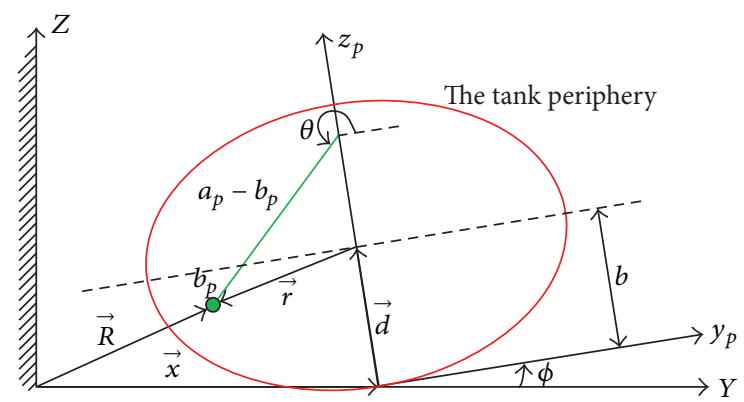

Figure 3: Analytical diagram for the motion of the trammel pendulum under the noninertial coordinate system.

$$
\begin{aligned}
\frac{m_{p}}{m}= & 0.7844-1.729 \Delta+0.3351 \Lambda+1.156 \Delta^{2}+0.7256 \Lambda \Delta \\
& -0.1254 \Lambda^{2}-0.3219 \Delta^{3}-0.9152 \Lambda \Delta^{2}+0.08043 \Lambda^{2} \Delta,
\end{aligned}
$$

$$
\begin{gathered}
m_{f}=m-m_{p}, \\
\frac{\left(b-b_{f}\right)}{b}=\frac{\left[m\left(b-b_{c g}\right)-m_{f}\left(b-b_{p}\right)\right]}{m_{f} b}
\end{gathered}
$$

where $\Delta$ is the liquid fill percentage, which is the ratio of the height of liquid's level surface to that of the tank, and $\Lambda$ is the ratio of the tank's width to its height.

The detailed derivation process for (1)-(5) is given by Zheng et al. [21].

According to Figure 1, the following equation holds:

$$
\frac{a}{b}=\frac{a_{\mathrm{cg}}}{b_{\mathrm{cg}}}=\frac{a_{p}}{b_{p}}=\Lambda .
$$

From (6), the arm length of the pendulum can be expressed as follows:

$$
a_{p}=b_{p} \times \Lambda
$$

As the mass of liquid bulk can easily be acquired while the tank's size and liquid density are given, the pendulum mass and the fixed liquid mass can be obtained using (3) and (4).

\subsection{Motion Equations of the Trammel Pendulum under the} Noninertial Coordinate System. During the driving process, the vehicle's driving state changes regularly under the influence of a variety of factors. It leads to the movement of the tank, and in turn the coordinate system is being fixed relative to the tank. Therefore, motion equations for a trammel pendulum under a noninertial coordinate system (translation and rotation are included) should be derived in order to study the motion characteristics of liquid bulk when tankers are being driven on curved sections of road, avoiding obstacles or changing lanes.

The schematic diagram for the motion of the tank when vehicle turns right is presented in Figure 3. For convenient analysis of the vehicle's roll stability, the origin of the tankfixed coordinate system is located at the bottom of the tank.
This location will not impact the motion analysis result. The origin of the earth-fixed coordinate system also is located at the bottom of the tank when the vehicle is static. $\vec{x}$ is the distance vector from the origin of the earth-fixed coordinate system to that of the tank-fixed coordinate system. $\vec{R}$ is the absolute position vector of the pendulum mass ball. $\vec{R}$ is the absolute position vector of the pendulum mass ball. $\phi$ is the tank roll angle, which is defined as the angle formed by rotating the $Y$-axis counterclockwise till the position of the $y_{p}$-axis.

With reference to Figure 3, the absolute position of the pendulum mass ball can be obtained as follows:

$$
\begin{aligned}
\vec{R}= & {\left[x-b \sin \phi+\left(a_{p} \cos \theta-b_{p} \sin \theta \tan \phi\right) \cos \phi\right] \vec{i} } \\
& +\left[b \cos \theta+\left(b_{p} \sin \theta+a_{p} \cos \theta \tan \phi\right) \cos \phi\right] \vec{j} .
\end{aligned}
$$

The velocity of the pendulum mass ball can be obtained by solving the first-order derivative of (8), which may be presented as follows:

$$
\begin{aligned}
\dot{R}=[\dot{x} & -\left(b \cos \phi+a_{p} \cos \theta \sin \phi+b_{p} \sin \theta \cos \phi\right) \dot{\phi} \\
- & \left.\left(a_{p} \sin \theta \cos \phi+b_{p} \cos \theta \sin \phi\right) \dot{\theta}\right] \vec{i} \\
+ & {\left[\left(-b \sin \phi-b_{p} \sin \theta \sin \phi+a_{p} \cos \theta \cos \phi\right) \dot{\phi}\right.} \\
& \left.+\left(b_{p} \cos \theta \cos \phi-a_{p} \sin \theta \sin \phi\right) \dot{\theta}\right] \vec{j} .
\end{aligned}
$$

Similarly, the acceleration of the pendulum mass ball can be acquired by solving the differential of (9), which may be written as follows:

$$
\begin{aligned}
\ddot{R}=[- & \ddot{\phi}\left(b \cos \phi+a_{p} \cos \theta \sin \phi+b_{p} \sin \theta \cos \phi\right) \\
- & \ddot{\theta}\left(a_{p} \sin \theta \cos \phi+b_{p} \cos \theta \sin \phi\right) \\
+ & 2 \dot{\theta} \dot{\phi}\left(a_{p} \sin \theta \sin \phi-b_{p} \cos \theta \cos \phi\right) \\
+ & \dot{\phi}^{2}\left(b \sin \phi-a_{p} \cos \theta \cos \phi+b_{p} \sin \theta \sin \phi\right) \\
+ & \left.\dot{\theta}^{2}\left(-a_{p} \cos \theta \cos \phi+b_{p} \sin \theta \sin \phi\right)+\ddot{x}\right] \vec{i} \\
+[ & \ddot{\phi}\left(-b \sin \phi+a_{p} \cos \theta \cos \phi-b_{p} \sin \theta \sin \phi\right) \\
& +\ddot{\theta}\left(b_{p} \cos \theta \cos \phi-a_{p} \sin \theta \sin \phi\right) \\
& -\dot{\theta}^{2}\left(a_{p} \cos \theta \sin \phi+b_{p} \sin \theta \cos \phi\right) \\
& +\dot{\phi}^{2}\left(-b \cos \phi-a_{p} \cos \theta \sin \phi-b_{p} \sin \theta \cos \phi\right) \\
& \left.-2 \dot{\theta} \dot{\phi}\left(a_{p} \sin \theta \cos \phi+b_{p} \cos \theta \sin \phi\right)\right] \vec{j} .
\end{aligned}
$$

The zero of potential energy is defined as the $Y$-axis, and the potential energy of the pendulum mass ball can be presented as follows:

$$
V=m g\left(b+b_{p} \sin \theta+a_{p} \cos \theta \tan \phi\right) \cos \phi .
$$


According to (10) and (11), a Lagrangian function can be used to obtain the motion equation of the pendulum system, which is written as follows:

$$
L=T-V,
$$

where $T$ is the kinetic energy of the pendulum mass ball.

The motion of the trammel pendulum system can be expressed by

$$
\frac{\partial}{\partial t}\left(\frac{\partial L}{\partial \dot{a}_{j}}\right)-\frac{\partial L}{\partial a_{j}}=0
$$

where $a_{j}$ is the degree of freedom for system $L$.

According to the motion analysis of the trammel pendulum, $\theta$, the pendulum amplitude, and $\phi$, the tank roll angle, are the two degrees of freedom for the trammel pendulum system.

The differential of (12) with respect to $\theta$ can be expressed as follows:

$$
\begin{aligned}
\frac{\partial L}{\partial \theta}=m[ & \dot{\phi}^{2}\left(-a_{p}^{2} \sin \theta \cos \theta+b_{p}^{2} \sin \theta \cos \theta+b_{p} b \cos \theta\right) \\
& +\dot{\theta}^{2}\left(a_{p}^{2} \sin \theta \cos \theta-b_{p}^{2} \sin \theta \cos \theta\right) \\
& +\dot{\theta} \dot{\phi}\left(a_{p} b \cos \theta\right) \\
& -\dot{x} \dot{\phi}\left(-a_{p} \sin \theta \sin \phi+b_{p} \cos \theta \cos \phi\right) \\
& -\dot{x} \dot{\theta}\left(a_{p} \cos \theta \cos \phi-b_{p} \sin \theta \sin \phi\right) \\
& \left.-g\left(b_{p} \cos \theta-a_{p} \sin \theta \tan \phi\right) \cos \phi\right] .
\end{aligned}
$$
by

The differential of (12) with respect to $\dot{\theta}$ can be expressed

$$
\begin{aligned}
& \frac{\partial L}{\partial \dot{\theta}}=m[ \dot{\theta}\left(a_{p}^{2} \sin ^{2} \theta+b_{p}^{2} \cos ^{2} \theta\right)+\dot{\phi}\left(a_{p} b_{p}+a_{p} b \sin \theta\right) \\
&\left.-\dot{x}\left(a_{p} \sin \theta \cos \phi+b_{p} \cos \theta \sin \phi\right)\right] .
\end{aligned}
$$

Finally, the differential of (15) with respect to $t$ can be expressed as follows:

$$
\begin{aligned}
\frac{\partial}{\partial t}\left(\frac{\partial L}{\partial \dot{\theta}}\right)=m[ & \ddot{\theta}\left(a_{p}^{2} \sin ^{2} \theta+b_{p}^{2} \cos ^{2} \theta\right) \\
& +\dot{\theta}^{2}\left(2 a_{p}^{2} \sin \theta \cos \theta-2 b_{p}^{2} \sin \theta \cos \theta\right) \\
& +\ddot{\phi}\left(a_{p} b_{p}+a_{p} b \sin \theta\right)+\dot{\phi} \dot{\theta} a_{p} b \cos \theta \\
& -\ddot{x}\left(a_{p} \sin \theta \cos \phi+b_{p} \cos \theta \sin \phi\right) \\
& -\dot{x} \dot{\theta}\left(a_{p} \cos \theta \cos \phi-b_{p} \sin \theta \sin \phi\right) \\
& \left.+\dot{x} \dot{\phi}\left(a_{p} \sin \theta \sin \phi-b_{p} \cos \theta \cos \phi\right)\right] .
\end{aligned}
$$

Substituting (14)-(16) into (13), we get

$$
\begin{aligned}
\ddot{\theta} & \left(a_{p}^{2} \sin ^{2} \theta+b_{p}^{2} \cos ^{2} \theta\right)+\ddot{\phi}\left(a_{p} b_{p}+a_{p} b \sin \theta\right) \\
& +\dot{\phi}^{2}\left[\frac{1}{2}\left(a_{p}^{2}-b_{p}^{2}\right) \sin 2 \theta-b_{p} b \cos \theta\right] \\
& +\frac{1}{2} \dot{\theta}^{2}\left(a_{p}^{2}-b_{p}^{2}\right) \sin 2 \theta \\
& -\ddot{x}\left(a_{p} \sin \theta \cos \phi+b_{p} \cos \theta \sin \phi\right) \\
& +g\left(b_{p} \cos \theta \cos \phi-a_{p} \sin \theta \sin \phi\right)=0
\end{aligned}
$$

The differential of (12) with respect to $\phi$ can be expressed as follows:

$$
\begin{aligned}
\frac{\partial L}{\partial \phi}=m & {\left[\dot{x} \dot{\phi}\left(b \sin \phi-a_{p} \cos \theta \cos \phi+b_{p} \sin \theta \sin \phi\right)\right.} \\
& -\dot{x} \dot{\theta}\left(-a_{p} \sin \theta \sin \phi+b_{p} \cos \theta \cos \phi\right) \\
& -g a_{p} \cos \theta \sec \phi \\
& \left.+g\left(b+b_{p} \sin \theta+a_{p} \cos \theta \tan \phi\right) \sin \phi\right] .
\end{aligned}
$$
by

The differential of (12) with respect to $\dot{\phi}$ can be expressed

$$
\begin{aligned}
\frac{\partial L}{\partial \dot{\phi}}=m & {\left[\dot{\phi}\left(b^{2}+a_{p}^{2} \cos ^{2} \theta+b_{p}^{2} \sin ^{2} \theta+2 b_{p} b \sin \theta\right)\right.} \\
& +\dot{\theta}\left(a_{p} b_{p}+a_{p} b \sin \theta\right) \\
& \left.-\dot{x}\left(b \cos \phi+a_{p} \cos \theta \sin \phi+b_{p} \sin \theta \cos \phi\right)\right] .
\end{aligned}
$$

According to (19), the differential of (19) with respect to $t$ can be obtained as follows:

$$
\begin{aligned}
\frac{\partial}{\partial t}\left(\frac{\partial L}{\partial \dot{\phi}}\right) & \\
=m & {\left[\ddot{\phi}\left(b^{2}+a_{p}^{2} \cos ^{2} \theta+b_{p}^{2} \sin ^{2} \theta+2 b_{p} b \sin \theta\right)\right.} \\
& +\ddot{\theta}\left(a_{p} b_{p}+a_{p} b \sin \theta\right)+a_{p} b \dot{\theta}^{2} \cos \theta \\
& +\dot{\phi} \dot{\theta}\left(-2 a_{p}^{2} \sin \theta \cos \theta+2 b_{p}^{2} \sin \theta \cos \theta+2 b_{p} b \cos \theta\right) \\
& +\dot{x} \dot{\theta}\left(a_{p} \sin \theta \sin \phi-b_{p} \cos \theta \cos \phi\right) \\
& +\dot{x} \dot{\phi}\left(b \sin \phi-a_{p} \cos \theta \cos \phi+b_{p} \sin \theta \sin \phi\right) \\
& \left.-\ddot{x}\left(b \cos \phi+a_{p} \cos \theta \sin \phi+b_{p} \sin \theta \cos \phi\right)\right] .
\end{aligned}
$$


Substituting (18)-(20) into (13) we get

$$
\begin{aligned}
\ddot{\phi}[ & \left.\left(b+b_{p} \sin \theta\right)^{2}+a_{p}^{2} \cos ^{2} \theta\right] \\
& +2 \dot{\theta} \dot{\phi}\left[\frac{1}{2} \sin 2 \theta\left(b_{p}^{2}-a_{p}^{2}\right)+b_{p} b \cos \theta\right] \\
& +\ddot{\theta}\left(a_{p} b_{p}+a_{p} b \sin \theta\right) \\
& -\ddot{x}\left(b \cos \phi+a_{p} \cos \theta \sin \phi+b_{p} \sin \theta \cos \phi\right) \\
& +\dot{\theta}^{2} a_{p} b \cos \theta \\
& -g\left[\left(b+b_{p} \sin \theta\right) \sin \phi-a_{p} \cos \theta \cos \phi\right]=0 .
\end{aligned}
$$

Equations (17) and (21) are the equations of motion of the trammel pendulum under the noninertia coordinate system, and the pendulum motion characteristics under the translation and rotation of the coordinate system can be analyzed using (17) and (21).

\section{Tank Truck Dynamic Model}

3.1. Assumptions. The roll stability of a tank truck is greatly influenced by transient liquid sloshing in partially filled tanks. Due to the flow characteristic of liquid bulk, transient liquid sloshing is produced when the vehicle's driving state changes, which leads to the oscillation of the pendulum mass ball. To simplify the analysis of the tank truck's roll stability, some assumptions are made and listed as follows.

(1) The sprung mass is the vehicle mass that lies over the suspension system, in which the mass of the liquid cargo is not included. In this paper, vehicle and liquid bulk, as the solid and fluid parts respectively, are not mixed with each other.

(2) In the transverse direction, the CG of the sprung mass is located at the bottom of the tank. Moreover, the sprung mass is symmetrical about the longitudinal axis of the vehicle.

(3) Transient liquid sloshing is merely produced along the transverse direction; longitudinal liquid sloshing is not taken into consideration. The movement and mass distribution of the liquid bulk are completely the same at different tank cross sections, which means that the CG of liquid bulk is located in the middle of the tank along the longitudinal direction.

(4) The liquid-free surface is not broken during the vehicle's driving process.

3.2. Force Analysis of the Tank Truck. Based on the assumptions given in Section 3.1, a reference coordinate system for tank truck dynamic analysis was selected first.

The coordinate system being fixed relative to the vehicle was chosen as the reference coordinate system for the dynamic analysis of the tank truck and the establishment of the vehicle's dynamic model. The origin of the coordinate system is the point where the vertical line that goes through the CG of the vehicle when it is static intersects with the vehicle's roll axis.

The coordinate system being fixed relative to the vehicle's sprung mass was defined as the $x-y-z$ system, where the $x$ axis is parallel to the longitudinal axis of the vehicle and points in the direction in which the vehicle is being driven, the $y$-axis is perpendicular to the $x$-axis and points to the left from the driver's perspective, and the $z$-axis is perpendicular to the $x y$ plane and points straight up.

The vehicle's unsprung mass is assumed not to produce roll movement.

The top view and the back view of the vehicle's dynamic analysis are plotted in Figures 4 and 5. According to Figure 5, the lateral inertial forces act on the sprung mass, the unsprung mass, the fixed liquid mass, and the pendulum mass, making up the vehicle's lateral inertial force. The tire cornering forces make up the external lateral force that acts on the vehicle according to Figure 4. On the basis of Newton's first law, when the vehicle is steady, its inertial lateral force and external lateral force are equal in value and positive in direction, which can be expressed as follows:

$$
\left(m_{t}+m_{f}\right) a_{s}+m_{p} a_{f}+m_{u} a_{u}=2\left(F_{f}+F_{r}\right)
$$

where $a_{s}$ is the lateral acceleration of sprung mass; $a_{u}$ is the lateral acceleration of unsprung mass; $a_{f}$ is the lateral acceleration of the pendulum mass ball. $m_{t}$ is sprung mass; $m_{u}$ is unsprung mass. $F_{f}$ is the front tire cornering force and $F_{r}$ is the rear tire cornering force.

Generally, the fixed liquid mass does not stay static but slides slowly with the aid of gravity while the tank tilts. However, the shift of the fixed liquid mass is quite small compared to that of the pendulum mass ball and its shift amount drops greatly with the increase in the liquid fill percentage. Therefore, the fixed liquid mass is assumed to be static and its position is assumed to stay constant as the tank rolls over. Based on this assumption, the fixed liquid mass can be seen as the solid part and its lateral acceleration is equal to that of the vehicle's sprung mass.

According to the dynamic analysis of the vehicle, the lateral acceleration of the sprung mass and unsprung mass can be presented by

$$
\begin{gathered}
a_{s}=V(\dot{\beta}+r)-h_{s} \ddot{\phi}+c \dot{r}, \\
a_{u}=V(\dot{\beta}+r)-e \dot{r},
\end{gathered}
$$

where $V$ is the vehicle's driving speed, $\beta$ the vehicle's slip angle, $r$ the vehicle's yaw angle, $h_{s}$ is the vertical distance from the CG of sprung mass to the roll center, $c$ is the longitudinal distance between the CG of sprung mass and that of the tank truck, and $e$ is the longitudinal distance between the CG of unsprung mass and that of the tank truck.

According to (10), the lateral acceleration of the pendulum mass ball can be written as follows:

$$
a_{f}=V \dot{\phi}-D_{1} \ddot{\phi}-D_{2} \ddot{\theta}+2 D_{3} \dot{\phi} \dot{\theta}+D_{4} \dot{\phi}^{2}+D_{5} \dot{\theta}^{2}
$$




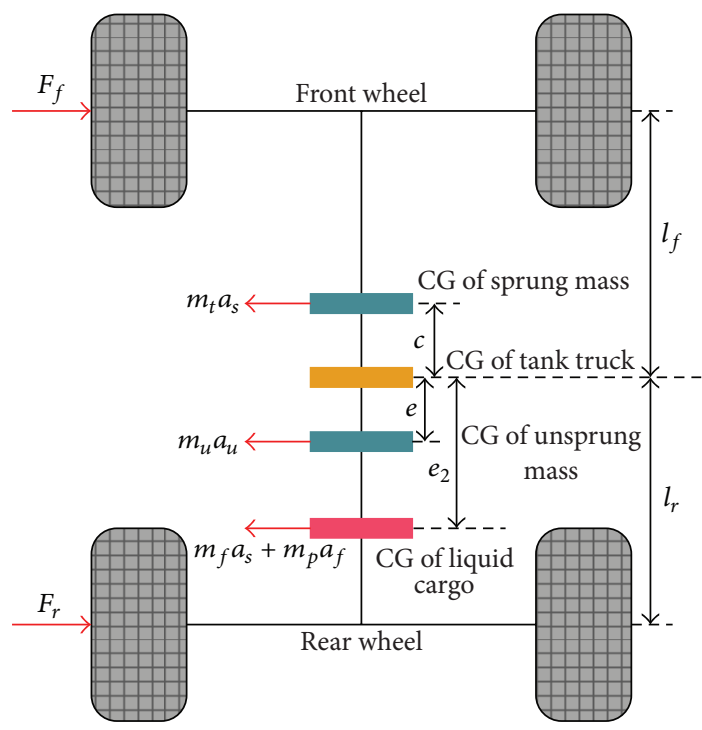

Figure 4: Top view of vehicle dynamic analysis.

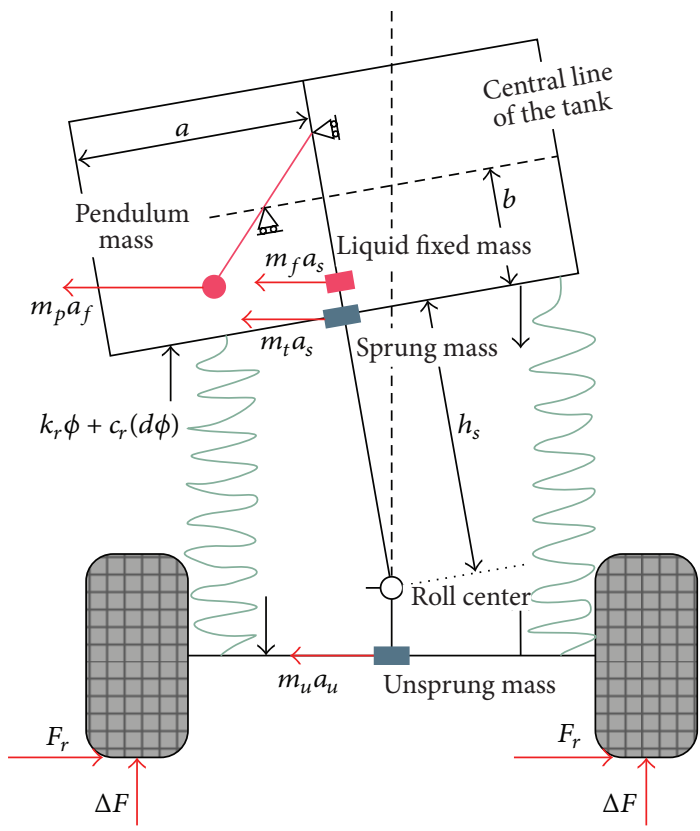

FIGURE 5: Back view of vehicle dynamic analysis.

where

$$
\begin{gathered}
D_{1}=b \cos \phi+a_{p} \cos \theta \sin \phi+b_{p} \sin \theta \cos \phi, \\
D_{2}=a_{p} \sin \theta \cos \phi+b_{p} \cos \theta \sin \phi, \\
D_{3}=a_{p} \sin \theta \sin \phi-b_{p} \cos \theta \cos \phi, \\
D_{4}=b \sin \phi-a_{p} \cos \theta \cos \phi+b_{p} \sin \theta \sin \phi, \\
D_{5}=-a_{p} \cos \theta \cos \phi+b_{p} \sin \theta \sin \phi .
\end{gathered}
$$

The force analysis in the roll plane is plotted in Figure 5. Due to the lateral inertia force, the sprung mass and the liquid

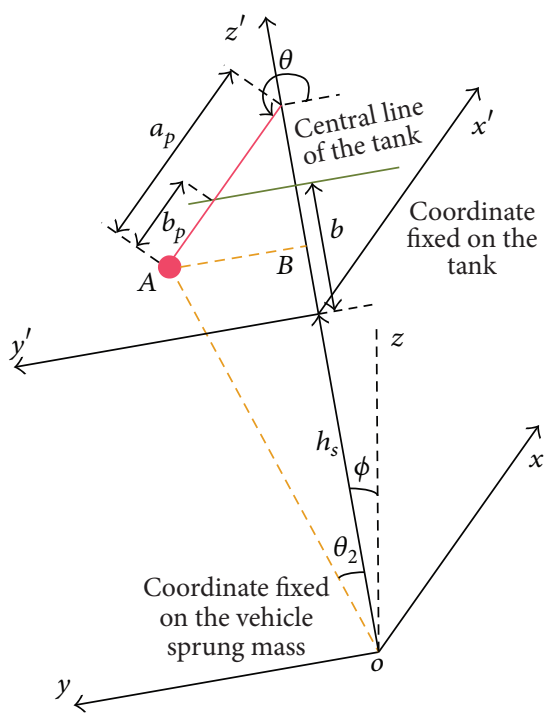

FIGURE 6: The schematic diagram for the pendulum rotating around the roll axis.

bulk rotate about the roll axis and bring about rollover torque. The roll angle about the roll axis for sprung mass and the fixed liquid mass is $\phi$, which can be seen from Figure 5. However, owing to the tank and the pendulum mass ball movement, the roll angle about the roll axis for the pendulum mass ball is composed of two parts, which is shown in Figure 6.

According to Figure 6, the roll angle about the roll axis for the pendulum mass ball can be expressed as follows:

$$
\angle=\phi+\theta_{2}
$$

where

$$
\theta_{2}=\arctan \left(\frac{A B}{B O}\right)=\arctan \left(\frac{-a_{p} \cos \theta}{h_{s}+b+b_{p} \sin \theta}\right) .
$$

Therefore, the absolute roll rate and roll angular acceleration can be obtained as follows based on (26):

$$
\dot{L}=\dot{\phi}+\dot{\theta}_{2} ; \quad \ddot{L}=\ddot{\phi}+\ddot{\theta}_{2},
$$

$$
\dot{\theta}_{2}=\frac{\dot{\theta} B_{1}}{B} ; \quad \ddot{\theta}_{2}=\frac{\left(A_{1} \ddot{\theta}-A_{2} \dot{\theta}^{2}\right)}{A} \text {, }
$$

where

$$
\begin{gathered}
B=\left(h_{s}+b+b_{p} \sin \theta\right)^{2}+\left(a_{p} \cos \theta\right)^{2} ; \\
B_{1}=a_{p}\left[\left(h_{s}+b\right) \sin \theta+b_{p}\right] ; \\
A=B^{2} ; \quad A_{1}=a_{p}\left[\left(h_{s}+b\right) \sin \theta+b_{p}\right] B ; \\
A_{2}=2 a_{p} \cos \theta\left[\left(h_{s}+b\right) \sin \theta+b_{p}\right] \\
\quad \times\left[b p\left(h_{s}+b+b_{p} \sin \theta\right)-a_{p}^{2} \sin \theta\right] \\
-a_{p} B\left(h_{s}+b\right) \cos \theta .
\end{gathered}
$$


On the basis of (28), the vehicle's roll balance equation and yaw balance equation can be acquired by differentiating the angular momentum with respect to time.

In general, the angular momentum of a rotating object is defined as the product of a body's rotational inertia tensor and rotational velocities about a particular coordinate.

(1) The Angular Momentum of Sprung Mass and the Fixed Liquid Mass. As the vehicle sprung mass and the fixed liquid mass have the same rotational velocity about the $x-y-z$ coordinate system, the angular momentum for the two parts is solved together.

According to the definition of angular momentum, that for the sprung mass and fixed liquid mass can be expressed as follows:

$$
H_{s}=\left[\begin{array}{ccc}
I_{x x s}+I_{x x f} & -I_{x y s}-I_{x y f} & -I_{x z s}-I_{x z f} \\
-I_{y x s}-I_{y x f} & I_{y y s}+I_{y y f} & -I_{y z s}-I_{y z f} \\
-I_{z x s}-I_{z x f} & -I_{z y s}-I_{z y f} & I_{z z s}+I_{z z f}
\end{array}\right]\left[\begin{array}{c}
\dot{\phi} \\
0 \\
r
\end{array}\right],
$$

where $I_{x x s}, I_{y y s}$, and $I_{z z s}$ are the moments of inertia for the sprung mass about the $x$-axis, $y$-axis, and $z$-axis, respectively; $I_{x x f}, I_{y y f}$, and $I_{z z f}$ are the moments of inertia for the fixed liquid mass about the $x$-axis, $y$-axis, and $z$-axis, respectively; $I_{x y s}=I_{y x s}, I_{x z s}=I_{z x s}$, and $I_{y z s}=I_{z y s}$ are the products of inertia for the sprung mass about the $x$ - and $y$-axis, $x$ - and $z$ axis, and $y$ - and $z$-axis, respectively; $I_{x y f}=I_{y x f}, I_{x z f}=I_{z x f}$, and $I_{y z f}=I_{z y f}$ are the products of inertia for the fixed liquid mass about the $x$ - and $y$-axis, $x$ - and $z$-axis, and $y$ - and $z$ axis, respectively.

According to the assumptions given in Section 3.1, the distribution of the vehicle's sprung mass is symmetrical about the $x z$-plane. Therefore, the products of inertia for the sprung mass can be expressed as follows:

$$
I_{x y s}=0 ; \quad I_{z y s}=0 .
$$

As the fixed liquid mass does not move while the tank tilts, the distribution of the fixed liquid mass is symmetrical about the $x_{t} z_{t}$-plane and the $x z$-plane. Therefore, the products of inertia for the fixed liquid mass can be expressed as follows:

$$
I_{x y f}=0 ; \quad I_{z y f}=0 .
$$

Therefore, based on (31)-(33), the angular momentum for the vehicle's sprung mass and the fixed liquid mass can be written as follows:

$$
\begin{aligned}
H_{s}= & {\left[\left(I_{x x s}+I_{x x f}\right) \dot{\phi}-\left(I_{x z s}+I_{x z f}\right) r\right] \vec{i} } \\
& +\left[-\left(I_{z x s}+I_{z x f}\right) \dot{\phi}+\left(I_{z z s}+I_{z z f}\right) r\right] \vec{j} .
\end{aligned}
$$

(2) The Angular Momentum of the Pendulum Mass. According to (28), the angular momentum of the pendulum mass can be expressed as follows:

$$
H_{p}=\left[\begin{array}{ccc}
I_{x x p} & -I_{x y p} & -I_{x z p} \\
-I_{y x p} & I_{y y p} & -I_{y z p} \\
-I_{z x p} & -I_{z y p} & I_{z z p}
\end{array}\right]\left[\begin{array}{c}
\dot{\phi}+\dot{\theta}_{2} \\
0 \\
r
\end{array}\right],
$$

where $I_{x x p}, I_{y y p}$, and $I_{z z p}$ are the moments of inertia for the pendulum mass about the $x$-axis, $y$-axis, and $z$-axis, respectively; $I_{x y p}=I_{y x p}, I_{x z p}=I_{z x p}$, and $I_{y z p}=I_{z y p}$ are the products of inertia for the pendulum mass about the $x$ - and $y$-axis, the $x$-and $z$-axis, and the $y$-and $z$-axis, respectively.

The expanded form of (35) is written as

$$
\begin{aligned}
H_{p}= & {\left[I_{x x p}\left(\dot{\phi}+\dot{\theta}_{2}\right)-I_{x z p} r\right] \vec{i}+\left[-I_{x y p}\left(\dot{\phi}+\dot{\theta}_{2}\right)-I_{y z p} r\right] \vec{j} . } \\
& +\left[-I_{x z p}\left(\dot{\phi}+\dot{\theta}_{2}\right)+I_{z z p} r\right] \vec{k} .
\end{aligned}
$$

(3) The Angular Momentum of the Unsprung Mass. The yaw movement is the only rotational degree of freedom for the unsprung mass. Therefore, the angular momentum of the unsprung mass can be obtained by multiplying together the moment of inertia about the $z$-axis and the yaw rate about that, which is written as follows:

$$
H_{u}=I_{z z u} r \vec{k}
$$

where $I_{z z u}$ is the moment of inertia about the $z$-axis for the unsprung mass.

The change in the vehicle fixed coordinate after it has been driving for time $\Delta t$ is plotted in Figure 7, from which we can see that the differentials of the $\vec{i}, \vec{j}$, and $\vec{k}$ unit vectors can be expressed as follows:

$$
\dot{\vec{i}}=r \vec{j} ; \quad \dot{\vec{j}}=-r \vec{i}+\dot{\phi} \vec{k} ; \quad \dot{\vec{k}}=-\dot{\phi} \vec{j} .
$$

The first-order derivatives of (34), (36), and (37) can be obtained using (38). Thus, the vehicle's roll moment and yaw moment have been acquired.

According to the moment balance between the vehicle inertia moment and the external moment, the vehicle's roll and yaw moment balance equation can be expressed as follows:

$$
\begin{aligned}
I_{z} \dot{r}- & I_{x z} \ddot{\phi}-m_{f} a_{s} e_{2}-m_{p} a_{f} e_{2}-I_{x z p}(\ddot{\phi}+\ddot{\theta})+I_{z z p} \dot{r} \\
& \quad I_{x y p}(\dot{\phi}+\dot{\theta})^{2}-I_{y z p} r(\dot{\phi}+\dot{\theta})=2\left(F_{f} l_{f}-F_{r} l_{r}\right), \\
I_{x} \ddot{\phi}- & I_{x z} \dot{r}+m_{t} h_{s} V(\dot{\beta}+r)+H_{1} m_{f} a_{s}+\left(h_{s}+b\right) m_{p} a_{f} \\
& +I_{x x p}(\ddot{\phi}+\ddot{\theta})-I_{x z p} \dot{r}+I_{x y p} r(\dot{\phi}+\dot{\theta})+I_{y z p} r^{2} \\
= & -k_{\phi} \phi-c_{\phi} \dot{\phi} \\
& +\phi\left(m_{t} g h_{s}+m_{f} g H_{1}+m_{p} g\left(h_{s}+b\right)\right)-m_{p} g a_{p} \cos \theta,
\end{aligned}
$$

where $l_{f}$ is the longitudinal distance from the CG of the tank truck to the front wheel, $l_{r}$ is the longitudinal distance from the CG of the tank truck to the rear wheel, $k_{\phi}$ is the suspension roll stiffness, $c_{\phi}$ is the suspension roll damping, $e_{2}$ is the longitudinal distance from the CG of the tank truck to the CG of liquid cargo, and $H_{1}$ is the vertical distance from the $C G$ of the fixed liquid mass to the roll center. 
Based on the parallel axis theorem of the moment of inertia, the following equation can be obtained:

$$
\begin{gathered}
I_{z}=I_{z z s}+I_{z z u}+m_{t} c^{2}+m_{u} e^{2}+I_{z z f}, \\
I_{x}=I_{x x s}+I_{x x f}+m_{t} h_{s}^{2}, \\
I_{z x}=I_{x z s}+I_{x z f}+m_{t} h_{s} c .
\end{gathered}
$$

The dynamic model of the tank truck is composed of (17) and (39)-(40).

The tire cornering force in the dynamic model can be obtained using the magic formula for tires, which is given by

$$
F=D \sin (C \arctan (B \alpha-E(B \alpha-\arctan B \alpha))),
$$

where $\alpha$ is the tire's sideslip angle, and $B, C, D$, and $E$ are the constant parameters of the magic formula for tires.

The tire sideslip angle in (41) can be expressed as follows:

$$
\begin{gathered}
\alpha_{f}=\arctan \left(\frac{V \beta+r l_{f}}{V}\right)-\delta_{f}, \\
\alpha_{r}=\arctan \left(\frac{V \beta-r l_{r}}{V}\right)-\delta_{r},
\end{gathered}
$$

where $\alpha_{f}$ is the front tire sideslip angle and $\alpha_{r}$ is the rear tire sideslip angle; $\delta_{f}$ is the front wheel steering angle and $\delta_{r}$ is the rear wheel steering angle.

For the convenience of vehicle dynamic simulation, the tank truck dynamic model is translated into the following form:

$$
M x=N
$$

and (43) can be rewritten as follows:

$$
\left[\begin{array}{llll}
m_{11} & m_{12} & m_{13} & m_{14} \\
m_{21} & m_{22} & m_{23} & m_{24} \\
m_{31} & m_{32} & m_{33} & m_{34} \\
m_{41} & m_{42} & m_{43} & m_{44}
\end{array}\right]\left[\begin{array}{c}
\dot{\beta} \\
\dot{r} \\
\ddot{\phi} \\
\ddot{\theta}
\end{array}\right]=\left[\begin{array}{l}
n_{1} \\
n_{2} \\
n_{3} \\
n_{4}
\end{array}\right],
$$

where

$$
\begin{gathered}
m_{11}=V\left(m_{t}+m_{f}+m_{p}+m_{u}\right), \\
m_{12}=c\left(m_{t}+m_{f}\right)-e m_{u}, \\
m_{13}=-h_{s}\left(m_{t}+m_{f}\right)-m_{p} D_{1}, \\
m_{14}=-m_{p} D_{2}, \\
m_{21}=V e_{2}\left(-m_{f}-m_{p}\right), \\
m_{22}=I_{z}-m_{f} c e_{2}+I_{z z p},
\end{gathered}
$$

$$
\begin{aligned}
& m_{23}=-I_{x z}+m_{f} h_{s} e_{2}+m_{p} D_{1} e_{2}-I_{x z p}, \\
& m_{24}=m_{p} D_{2} e_{2}-\frac{I_{x z p} A_{1}}{A}, \\
& m_{31}=V\left(m_{t} h_{s}+m_{f} H_{1}+m_{p} H\right) \text {, } \\
& m_{32}=-I_{x z}+m_{f} c H_{1}-I_{x z p}, \\
& m_{33}=I_{x}-m_{f} h_{s} H_{1}-m_{p} H D_{1}+I_{x x p} \text {, } \\
& m_{34}=\frac{I_{x x p} A_{1}}{A}-m_{p} H D_{2} \text {, } \\
& m_{41}=V\left(a_{p} \sin \theta \cos \phi+b_{p} \cos \theta \sin \phi\right) \text {, } \\
& m_{42}=0 \text {, } \\
& m_{43}=a_{p} b_{p}+a_{p} b \sin \theta \text {, } \\
& m_{44}=a_{p}^{2} \sin ^{2} \theta+b_{p}^{2} \cos ^{2} \theta, \\
& n_{1}=2\left(F_{f}+F_{r}\right)-r V\left(m_{t}+m_{f}+m_{u}\right) \\
& -m_{p}\left(2 D_{3} \dot{\theta} \dot{\phi}+D_{4} \dot{\phi}^{2}+D_{5} \dot{\theta}^{2}\right) \text {, } \\
& n_{2}=2\left(F_{f} l_{f}-F_{r} l_{r}\right)+m_{f} e_{2} V r \\
& +m_{p} e_{2}\left(2 D_{3} \dot{\theta} \dot{\phi}+D_{4} \dot{\phi}^{2}+D_{5} \dot{\theta}^{2}\right) \\
& -\frac{I_{x z p} \dot{\theta}^{2} A_{2}}{A}+I_{x y p}\left(\dot{\phi}+\frac{\dot{\theta} B_{1}}{B}\right)^{2} \\
& +I_{y z p} r\left(\dot{\phi}+\frac{\dot{\theta} B_{1}}{B}\right), \\
& n_{3}=-c_{\phi} \dot{\phi}+\phi\left(-k_{\phi}+m_{t} g h_{s}+m_{f} g H_{1}+m_{p} g H\right) \\
& -a_{p} m_{p} g \cos \theta-V r\left(m_{t} h_{s}+m_{f} H_{1}\right) \\
& -H m_{p}\left(2 D_{3} \dot{\theta} \dot{\phi}+D_{4} \dot{\phi}^{2}+D_{5} \dot{\theta}^{2}\right) \\
& -I_{x y p} r(\dot{\phi}+\dot{\theta})-r^{2} I_{y z p}+\frac{\dot{\theta}^{2} I_{x x p} A_{2}}{A}, \\
& n_{4}=-g\left(b_{p} \cos \theta \cos \phi-a_{p} \sin \theta \sin \phi\right) \\
& -\frac{1}{2} \dot{\theta}^{2}\left(a_{p}^{2}-b_{p}^{2}\right) \sin 2 \theta \\
& -\dot{\phi}^{2}\left[\frac{1}{2}\left(a_{p}^{2}-b_{p}^{2}\right) \sin 2 \theta-b_{p} b \cos \theta\right] .
\end{aligned}
$$

According to the Cramer rule, the variables in (43) can be expressed as follows while the matrix $M$ has a nonzero determinant:

$$
\begin{array}{ll}
x_{1}=\frac{M_{1}}{N}, & x_{2}=\frac{M_{2}}{N}, \\
x_{3}=\frac{M_{3}}{N}, & x_{4}=\frac{M_{4}}{N},
\end{array}
$$




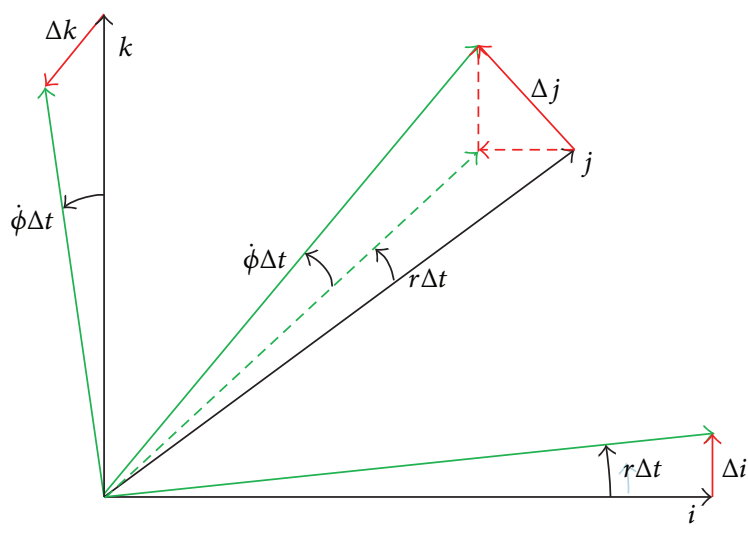

FIGURE 7: The change of the $x-y-z$ coordinate system.

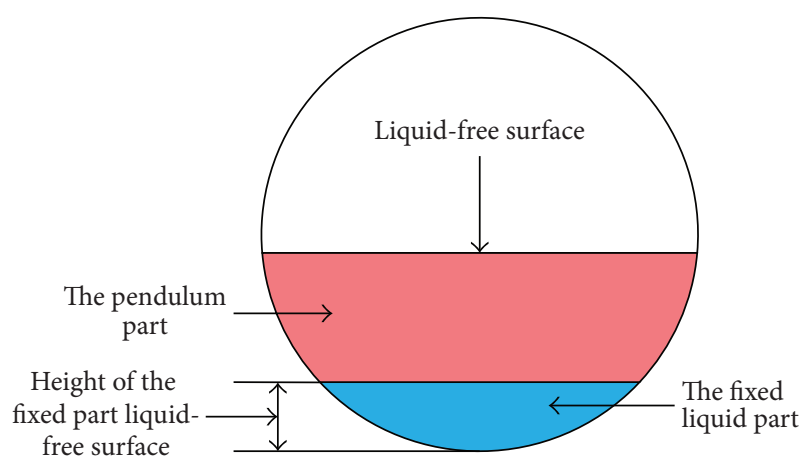

Figure 8: Schematic diagram for the division into the pendulum part and the fixed liquid part.

where $M_{i}, i=1,2,3,4$ is the matrix formed by replacing the $i$ th column of $M$ by the column vector $N$.

Based on (46), MATLAB's ODE algorithm is used to solve the differential equations of the vehicle's dynamic model.

3.3. The Inertia Tensor of the Pendulum Mass and the Fixed Liquid Mass. Before solving the differential equations of the vehicle's dynamic model, the inertia tensor for the fixed liquid mass and the pendulum mass are needed.

It is known that the liquid-free surface can be approximately described by a tilted straight line when transient liquid sloshing occurs. This means that the inertia tensor for the liquid bulk can be obtained. However, the obtained values are the sum of the pendulum part and the fixed liquid part. As it is quite difficult to divide liquid bulk physically into the pendulum part and the fixed liquid part, the fixed liquid part is assumed to locate at the bottom of the liquid bulk (as shown in Figure 8) and the height of the fixed liquid-free surface can be acquired using (3) and (4), while the density of the liquid cargo is already given. Based on this assumption, the inertia tensor for the fixed liquid part can be easily obtained. Hence, the inertia tensor for the pendulum part can also be obtained.

According to a market survey, the cross-sectional area of cylindrical tanks is usually close to $2.4 \mathrm{~m}^{2}$. HT5250GYQ3C, a typical tank truck of the HongTu brand, is chosen as the simulation object in this paper. The radius of the tank cross section is $1.8 \mathrm{~m}$ and the thickness of the tank walls is neglected. The length of the tank is $9 \mathrm{~m}$. The liquid cargo is assumed to be water, whose density is $1000 \mathrm{~kg} / \mathrm{m}^{3}$. CAD is used to calculate the inertia tensors for the pendulum mass and the fixed liquid mass as the liquid fill level changes from 0.1 to 0.9 with a 0.1 step size and as the tilt angle of the liquidfree surface changes from 0 degrees to 90 degrees with a 10 degree step size. The calculation results for liquid fill levels of $0.2,0.5$, and 0.8 are given in Tables 1,2 , and 3 .

As the fixed liquid part is assumed to be static as the tank tilts, the inertia tensor of the fixed liquid mass is always constant.

Polynomials are fitted for the data points listed in Tables 1,2 , and 3 , respectively, to obtain equations that describe the inertia tensor as a function of the liquid fill percentage and the liquid-free surface tilt angle. For arbitrary tilt angle of liquidfree surface under constant liquid fill level, interpolation is used to obtain the inertia tensor of the pendulum mass.

\section{Results and Discussion}

4.1. Tank Truck's Driving Stability When the Liquid Fill Level Is 0.5. A MATLAB simulation is carried out for the steering angle step test of the HongTu tank truck with a driving speed of $90 \mathrm{~km} / \mathrm{h}$ and a liquid fill level of 0.5 . The simulation results for different driving variables are plotted in Figure 9. Simulation results for a normal truck that has the same parameter values, load situation (i.e., 50\% laden), and driving velocity are plotted in Figure 10.

It is quite obvious that the vehicle's roll stability is greatly influenced by transient liquid sloshing in a partially filled tank. While the curves of the driving variables for the normal truck quickly return to the steady state, those for the tank truck fluctuate up and down for about $40 \mathrm{~s}$ before becoming steady, and the overshoot is quite large. However, the overshoot dampens quickly with the passage of time, and the damping frequency is close to the pendulum oscillation frequency. It can also be seen that the bigger the steering angle is, the more significant the effect on vehicle roll stability given by transient liquid sloshing is. As can be seen in Figure 9(d), the maximum roll rate of the tank truck is $0.2 \mathrm{rad} / \mathrm{s}$ while the steering angle is $0.02 \mathrm{rad}$, this value increases to $0.7 \mathrm{rad} / \mathrm{s}$ when the steering angle is $0.05 \mathrm{rad}$.

When the steering angle is $0.05 \mathrm{rad}$, the pendulum's amplitude changes from its original value of $4.71 \mathrm{rad}(270$ degrees) to $5.09 \mathrm{rad}$ (291.7 degrees) and stays steady with the aid of lateral acceleration and gravity. The movement of the pendulum mass ball increases the vehicle's rollover torque and results in a bigger roll angle for the tank truck (Figure 9(c)) compared to the normal truck (Figure 10(c)).

As the maximum roll rate of the vehicle is already quite large even though the steering angle is small, it seems that the vehicle may encounter rollover. However, as seen in Figure 9, the tank truck does not experience rollover but returns to a steady state after about $50 \mathrm{~s}$ of fluctuation, which means that whether or not the vehicle loses roll stability mostly depends on the duration of its transient response. 
TABLE 1: The inertia tensors of the pendulum mass and the fixed liquid mass when the liquid fill level is 0.2 .

\begin{tabular}{lcccccccc}
\hline & $I_{x x p}$ & $I_{z z p}$ & $I_{x y p}$ & $I_{x z p}$ & $I_{y z p}$ & $I_{x x f}$ & $I_{z z f}$ & $I_{x z f}$ \\
\hline $0^{\circ}$ & 1831 & 18574 & 0 & 597 & 0 & 215 & 4088 \\
$10^{\circ}$ & 1926 & 18613 & 117 & 607 & 338 & 215 & 4088 \\
$20^{\circ}$ & 2208 & 18711 & 230 & 637 & 684 & 215 & 4088 \\
$30^{\circ}$ & 2670 & 18871 & 336 & 687 & 1044 & 215 & 4088 \\
$40^{\circ}$ & 3298 & 19075 & 432 & 754 & 1420 & 215 & 4088 \\
$50^{\circ}$ & 4071 & 19283 & 515 & 837 & 1806 & 215 & 4088 \\
$60^{\circ}$ & 4967 & 19485 & 582 & 933 & 2192 & 215 & 401 \\
$70^{\circ}$ & 5959 & 19650 & 632 & 1039 & 2558 & 215 & 101 \\
$80^{\circ}$ & 7020 & 19770 & 662 & 1153 & 2883 & 215 & 4088 \\
$90^{\circ}$ & 8101 & 19783 & 672 & 1268 & 3135 & 215 & 4088 \\
\hline
\end{tabular}

TABLE 2: The inertia tensors of the pendulum mass and the fixed liquid mass when the liquid fill level is 0.5.

\begin{tabular}{lccccccc}
\hline & $I_{x x p}$ & $I_{z z p}$ & $I_{x y p}$ & $I_{x z p}$ & $I_{y z p}$ & $I_{x x f}$ & $I_{z z f}$ \\
\hline $0^{\circ}$ & 10837 & 45002 & 0 & 2285 & 0 & 3998 & 35642 \\
$10^{\circ}$ & 11023 & 45002 & 228 & 2305 & 1063 & 3998 & 35642 \\
$20^{\circ}$ & 11575 & 45002 & 449 & 2364 & 2094 & 3998 & 35642 \\
$30^{\circ}$ & 12477 & 45002 & 656 & 2461 & 3062 & 3998 & 35642 \\
$40^{\circ}$ & 13702 & 45002 & 843 & 2592 & 3936 & 3998 & 35642 \\
$50^{\circ}$ & 15212 & 45002 & 1005 & 2754 & 4691 & 3998 & 35642 \\
$60^{\circ}$ & 16960 & 45002 & 1136 & 2941 & 5303 & 3998 & 35642 \\
$70^{\circ}$ & 18895 & 45002 & 1233 & 3149 & 5754 & 3998 & 1212 \\
$80^{\circ}$ & 20957 & 45002 & 1292 & 3370 & 6031 & 3998 & 1212 \\
$90^{\circ}$ & 23084 & 45002 & 1312 & 3598 & 6124 & 39942 & 35642 \\
\hline
\end{tabular}

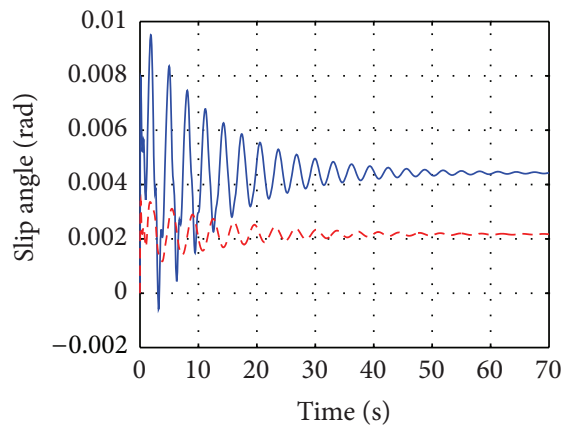

(a) Curves of slip angle

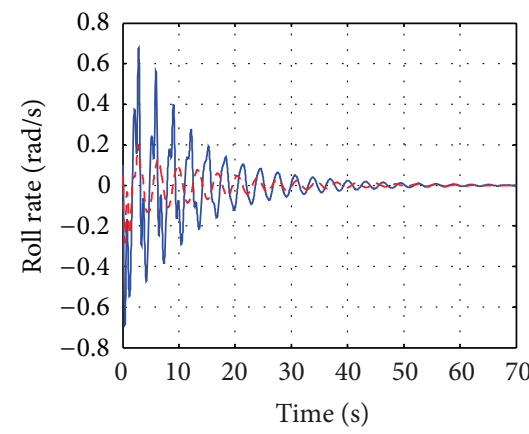

- Steering angle $=0.05 \mathrm{rad}$
- Steering angle $=0.02 \mathrm{rad}$

(d) Curves of roll rate

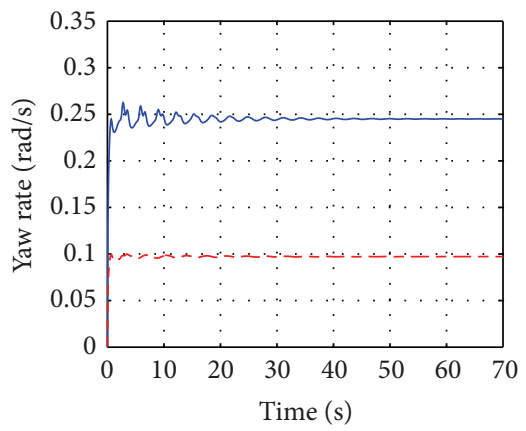

(b) Curves of yaw rate

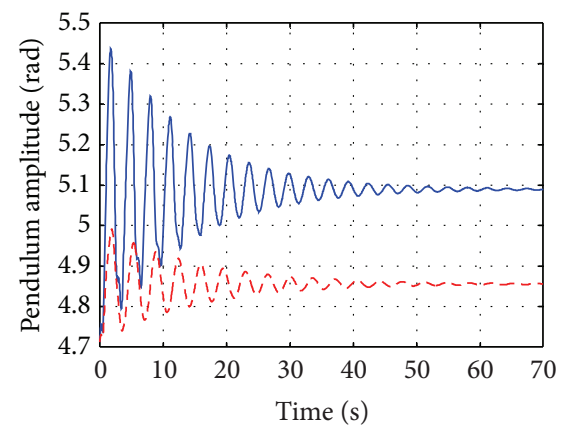

- Steering angle $=0.05 \mathrm{rad}$

- - Steering angle $=0.02 \mathrm{rad}$

(e) Curves of pendulum amplitude

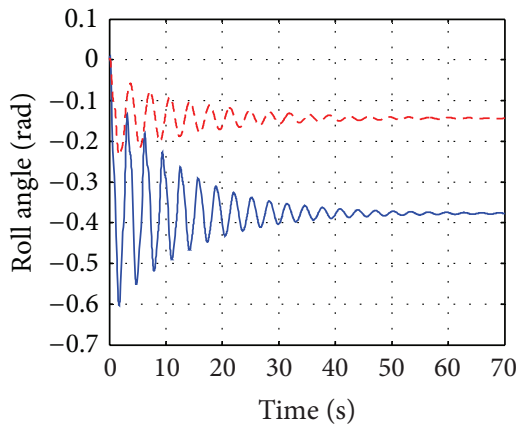

(c) Curves of roll angle

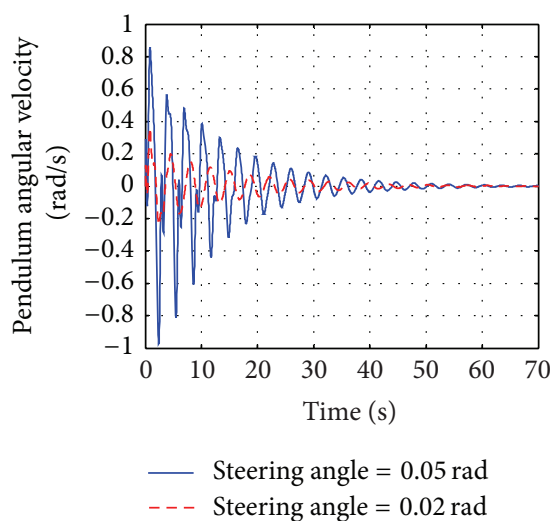

(f) Curves of pendulum angular velocity

FIGURE 9: Truck driving stability when liquid fill level is 0.5 . 
TABLE 3: The inertia tensors of the pendulum mass and the fixed liquid mass when the liquid fill level is 0.8 .

\begin{tabular}{lcccccccc}
\hline & $I_{x x p}$ & $I_{z z p}$ & $I_{x y p}$ & $I_{x z p}$ & $I_{y z p}$ & $I_{x x f}$ & $I_{z z f}$ \\
\hline $0^{\circ}$ & 16269 & 34190 & 0 & 2570 & 0 & 23308 & 104440 \\
$10^{\circ}$ & 16363 & 34150 & 117 & 2579 & 751 & 23308 & 104440 \\
$20^{\circ}$ & 16644 & 34040 & 230 & 2610 & 1461 & 23308 & 104440 \\
$30^{\circ}$ & 17107 & 33880 & 336 & 2659 & 2091 & 23308 & 104440 & 5008 \\
$40^{\circ}$ & 17732 & 33680 & 432 & 2726 & 2611 & 23308 & 104440 \\
$50^{\circ}$ & 18506 & 33470 & 515 & 2809 & 2998 & 23308 & 104440 \\
$60^{\circ}$ & 19400 & 33270 & 582 & 2905 & 3240 & 23308 & 5008 \\
$70^{\circ}$ & 20387 & 33100 & 632 & 3010 & 3337 & 23308 & 5008 \\
$80^{\circ}$ & 21438 & 32980 & 662 & 3122 & 3298 & 23308 & 104440 \\
$90^{\circ}$ & 22539 & 32980 & 672 & 3241 & 3135 & 23308 & 104440 \\
\hline
\end{tabular}

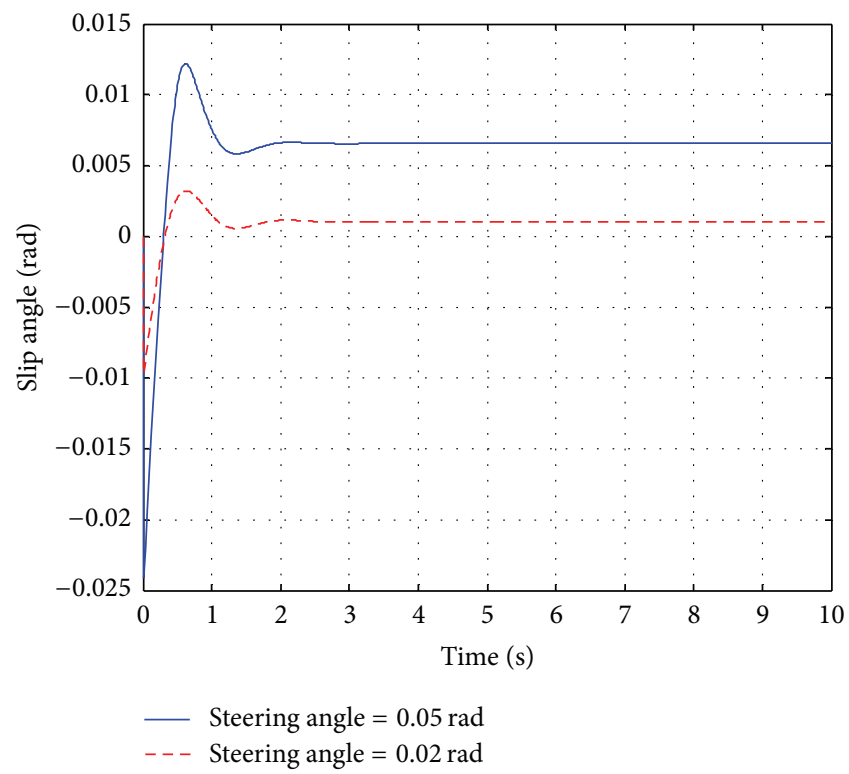

(a) Curves of slip angle

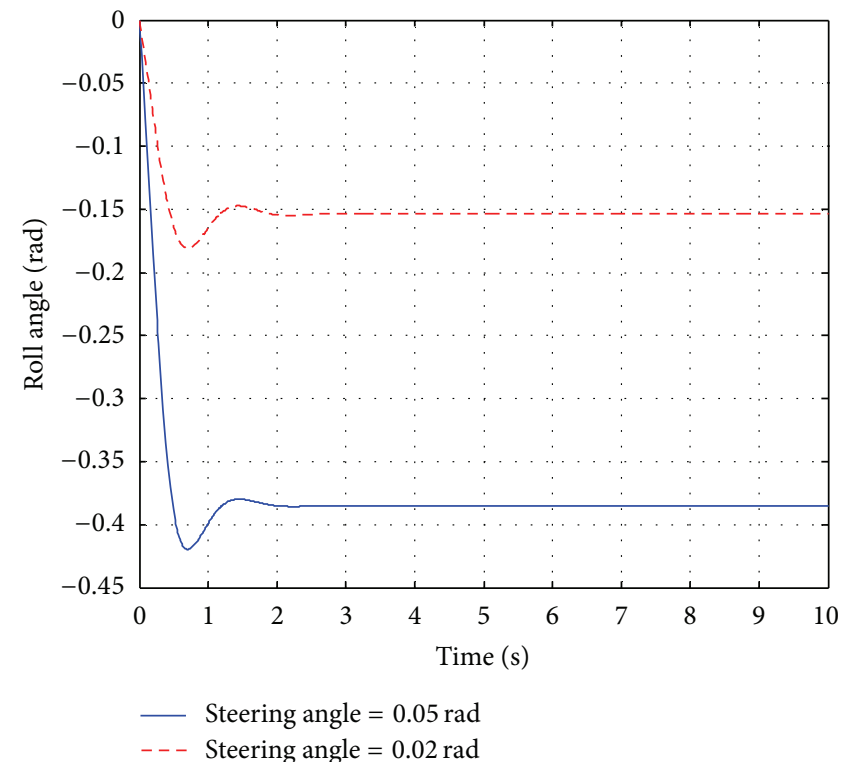

(c) Curves of roll angle

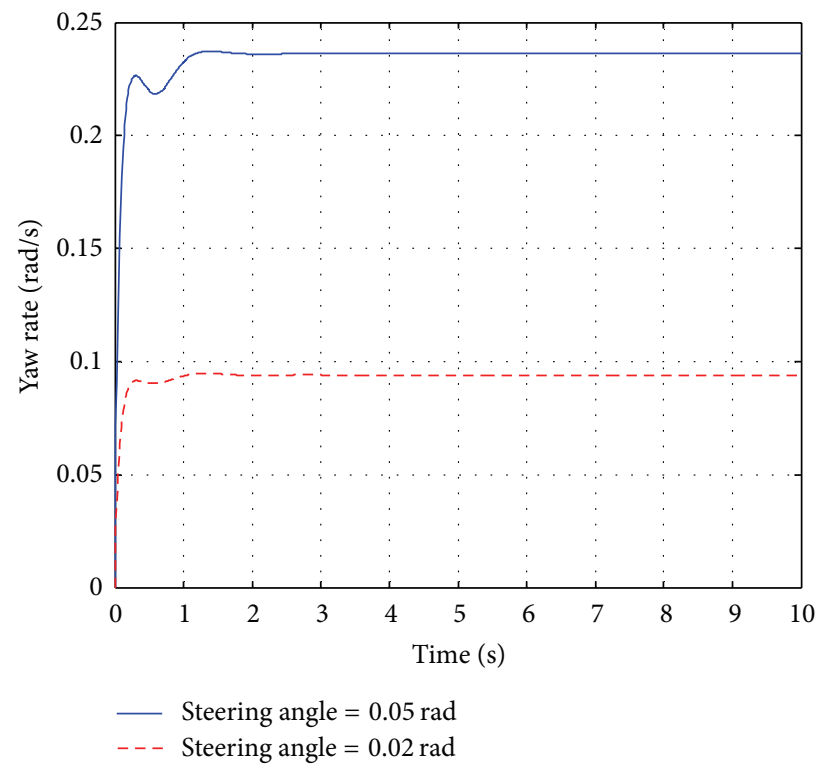

(b) Curves of yaw rate

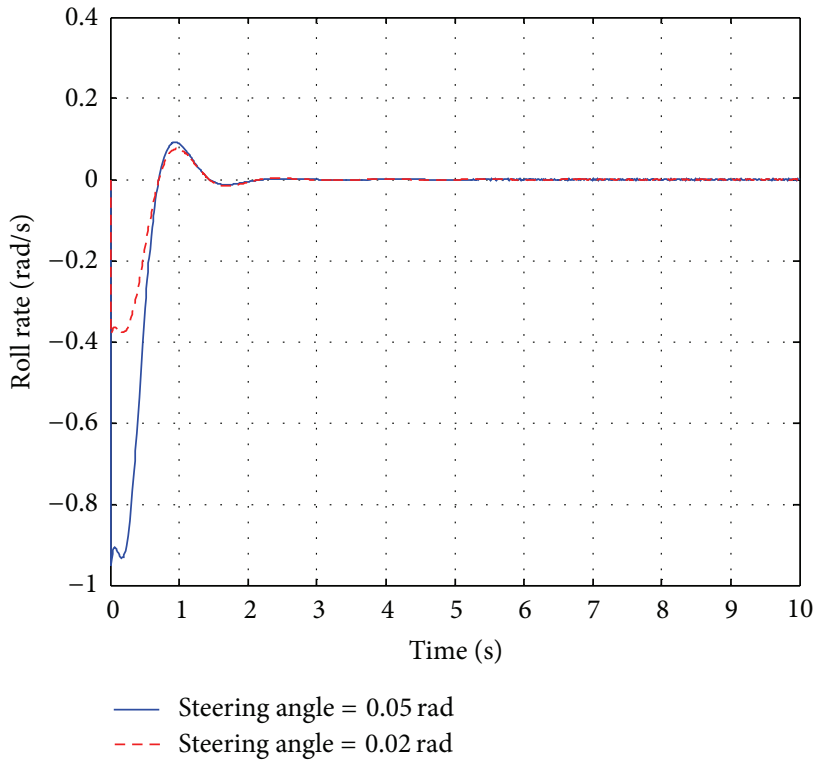

(d) Curves of roll rate

FIGURE 10: Driving stability of normal truck with $50 \%$ laden situation. 


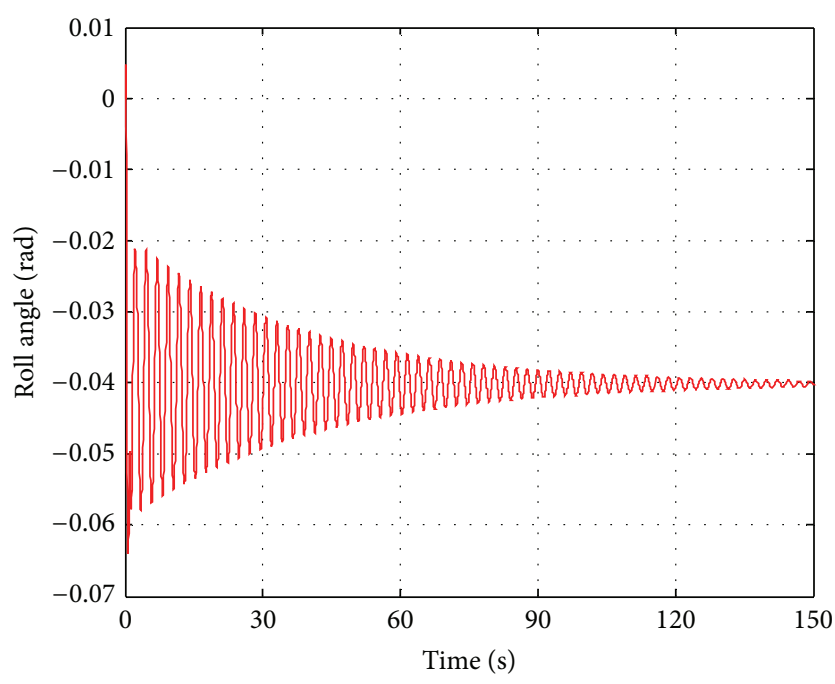

(a) Curves of roll angle

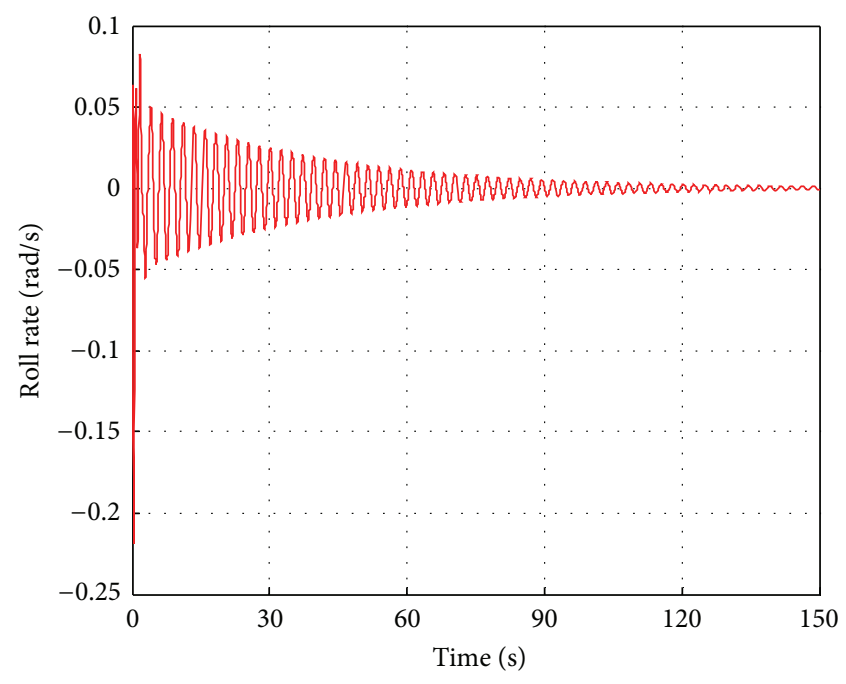

(b) Curves of roll rate

FIGURE 11: Tank truck driving stability when liquid fill level is 0.2 .

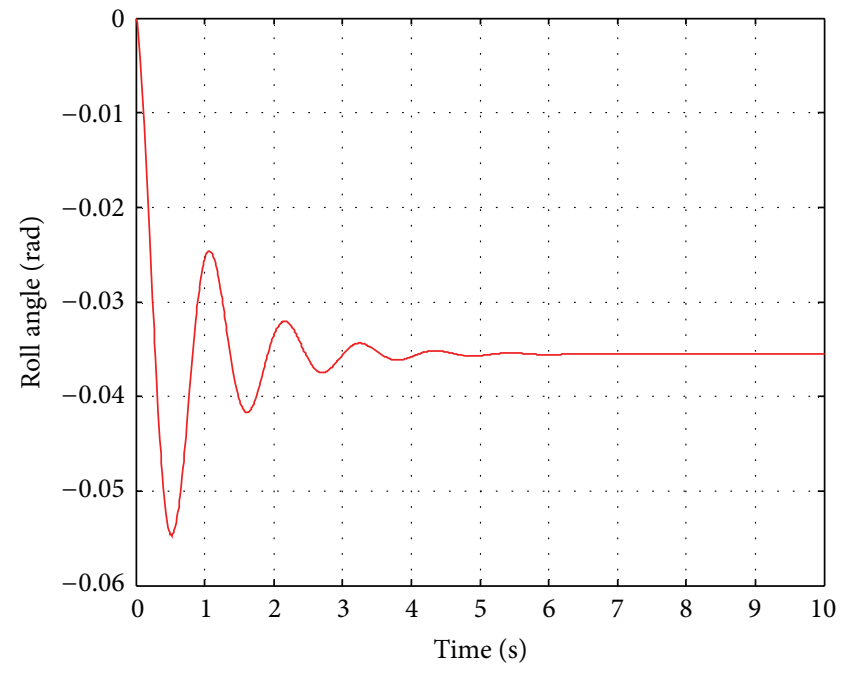

(a) Curves of roll angle

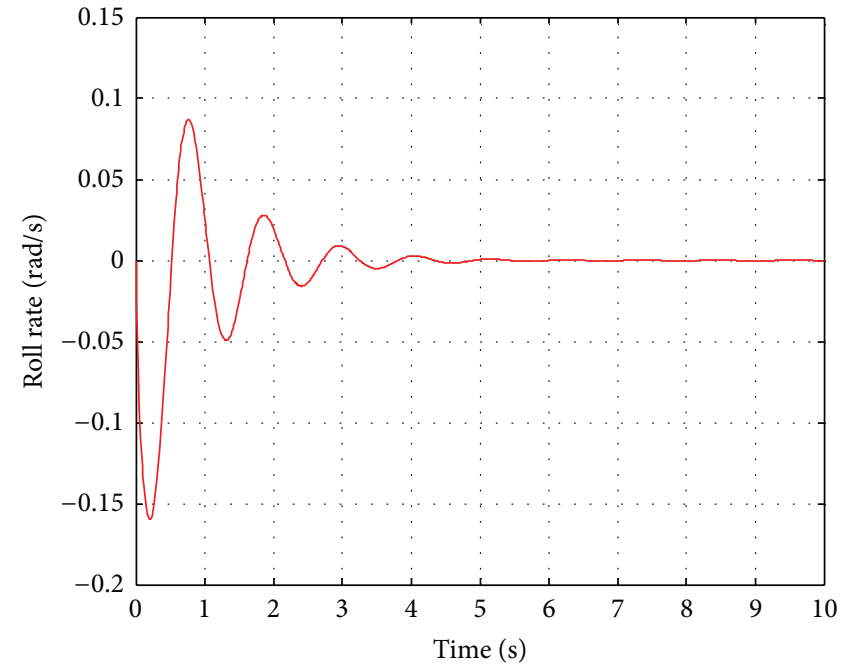

(b) Curves of roll rate

FIGURE 12: Driving stability of normal truck that is $20 \%$ laden.

From the comparison of Figures 9 and 10, it can also be seen that, although the overshoots of the driving variables of the tank truck are much bigger than those of the normal truck, the difference between their steady values is not so big, which means that the transient response of the tank truck is greatly affected by liquid sloshing, while the steady response is only slightly influenced by it.

4.2. Tank Truck's Driving Stability When the Liquid Fill Levels Are 0.2 and 0.8. To investigate the impact of the liquid fill level on the vehicle's roll stability, tank vehicles with liquid fill levels of 0.2 and 0.8 , with a steering angle of $0.02 \mathrm{rad}$, and with a driving speed of $90 \mathrm{~km} / \mathrm{h}$ are studied in a steering angle step test. The simulation results for the roll angle and roll rate are plotted in Figures 11 and 13.

At the same time, the roll stability of normal trucks with the same steering angle, parameter values, load situations, and driving speed are also investigated to determine the influence of transient liquid sloshing in a partially filled tank on vehicle roll stability. The simulation results for the roll angle and roll rate for the normal trucks are presented in Figures 12 and 14.

As can be seen from Figure 11, when the liquid fill level is 0.2 , the vehicle takes about $120 \mathrm{~s}$ to come to a steady state. The fluctuation times of the vehicle driving variables are much bigger than that when the liquid fill level was 0.5, as can be seen by comparing Figures 9 and 11. However, 


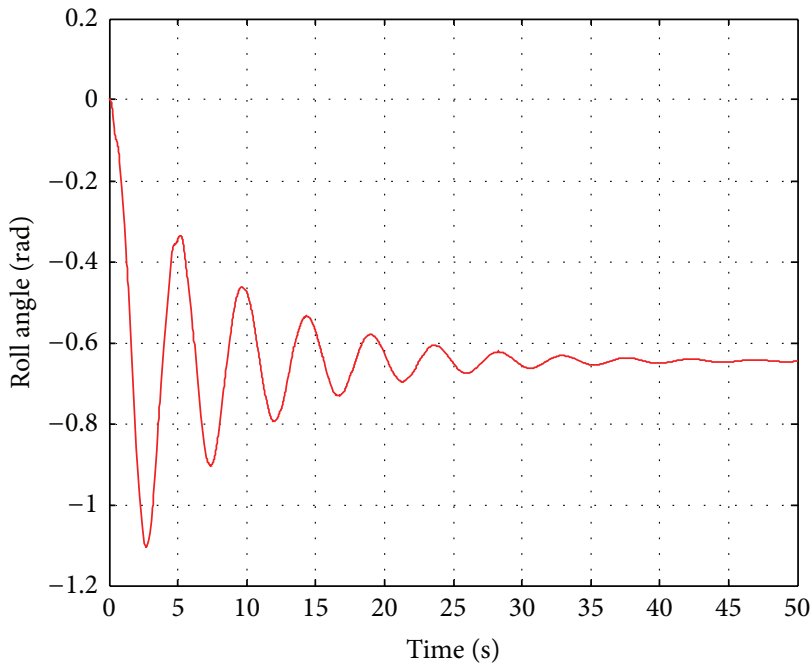

(a) Curves of roll angle

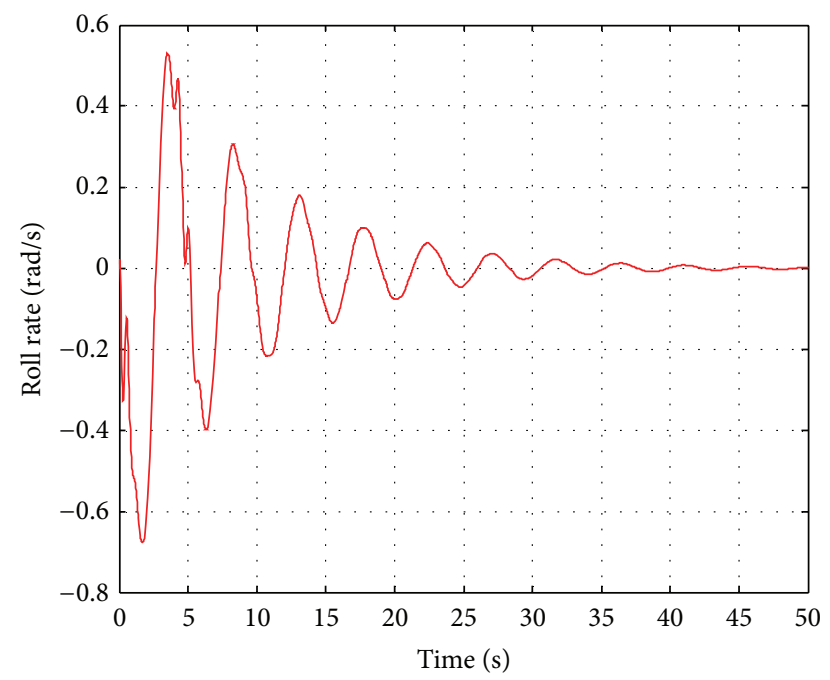

(b) Curves of roll rate

FIGURE 13: Tank truck driving stability when liquid fill level is 0.8 .

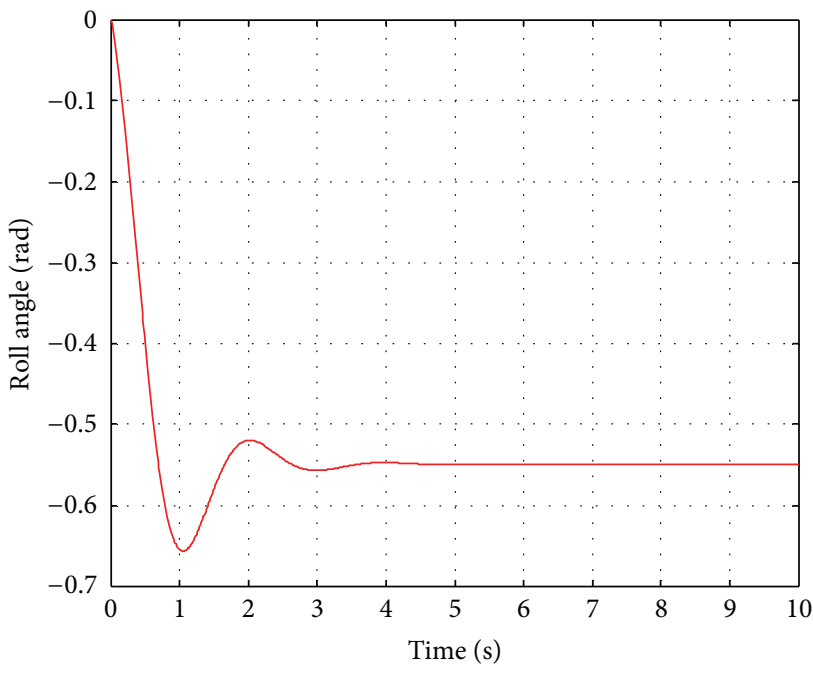

(a) Curves of roll angle

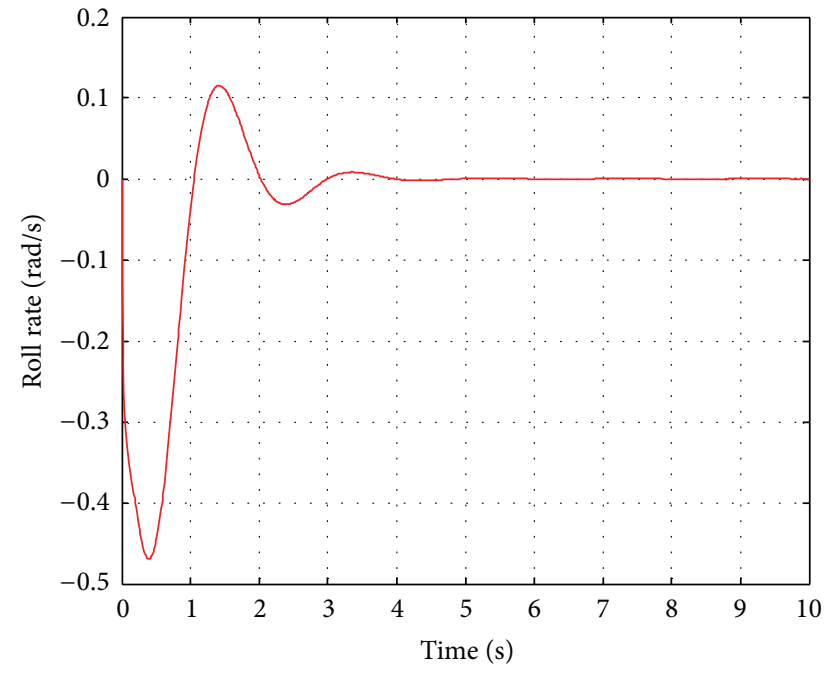

(b) Curves of roll rate

FIGURE 14: Driving stability of normal truck that is $80 \%$ laden.

the oscillation amplitude is reduced when the liquid fill level is lower. This is because when the liquid fill level is lower and the pendulum mass is much smaller, even though the arm length of the pendulum is bigger. It can be deduced that the oscillation amplitude is determined by the liquid sloshing mass to a large degree. This conclusion can also be proved by comparing Figures 9 and 13. Although the liquid fill level of 0.8 is bigger than that of 0.5 shown in Figure 9, the sloshing liquid mass for the former is smaller than that for the latter, as seen from (3) and (4). Therefore, vehicle roll stability when the liquid fill level is 0.5 is worse than that when the liquid fill level is 0.8 .

Above all, the tank vehicle's roll stability is greatly influenced when the liquid fill level is near to 0.5 , in which case the sloshing liquid mass occupies a large proportion of the liquid bulk and the mass of the liquid bulk is quite large. In this situation, the tank vehicle's roll stability is much worse than that of normal cars. However, when the liquid fill level is either fairly small or fairly large, the tank vehicle's roll stability is only slightly influenced by transient liquid sloshing. Therefore, a liquid fill level of $0.4-0.6$ is the worst load situation for tankers and should be avoided if possible.

\section{Conclusions}

To investigate the driving stability of tank trucks, the paper used an equivalent trammel pendulum for liquid sloshing in a partially filled tank. The trammel pendulum oscillated in the tank and was described in reference to tank-fixed coordinates. To couple the motion of the trammel pendulum with 
that of the tank truck, the motion equation of the pendulum under a noninertial coordinate system was analyzed using a Lagrangian function. Based on this, a dynamic model for a tank truck was established using Newton's first law and the angular momentum. A typical tank truck was selected and its driving stability is studied under a steering angle step test. Studying tankers' driving stability and the main influencing factors is of great importance for evaluating tankers' driving safety, as well as for developing active/passive roll control systems for them.

The following important discoveries were found from the driving stability simulations.

(1) The curves of the tank truck driving variables fluctuate until they return to the steady state. The fluctuation frequency descends with an increase in the liquid fill level while holding the testing situation constant. The fluctuation frequency is close to the pendulum's oscillation frequency. The overshoots of the driving variables depend on the pendulum mass, which is equal to the liquid mass that participates in transient sloshing.

(2) The pendulum mass occupies a large proportion of the mass of liquid bulk when the liquid fill level is in the range of 0.3-0.7. Also, the mass of liquid bulk is quite large in such cases, the overshooting of the tanker driving variables is quite large, and the tanker's driving stability is greatly impacted by transient liquid sloshing. Therefore, overshoot control for tankers' driving variables must be considered to support their driving safety. Moreover, the situation of a liquid fill level near to 0.5 is the worst state in terms of load and should be avoided.

(3) The driving stability of a tanker depends on the duration of the driving variables to a large degree.

Since we made the assumption in deriving the inertia tensors for the pendulum mass and the fixed liquid mass that the fixed mass is located at the bottom of the tank and is always static when the tank tilts, the inertia tensor value differs from the real situation, and the simulation results for the tank truck's driving stability will also have been slightly influenced. Thus, a theory and method for physically distinguishing the pendulum's mass and the fixed liquid mass from the liquid bulk will be proposed in a future study.

\section{Conflict of Interests}

The authors declare that there is no conflict of interests regarding the publication of this paper.

\section{Acknowledgments}

This research is supported by Research on Evaluation and Detection Technology of Handling and Driving Stability for Commercial Vehicles (no. 2009BAG13A04) and the National Natural Science Foundation of China (Grant nos. 51375200 and 51208225).

\section{References}

[1] J. Woodrooffe, "Evaluation of dangerous goods vehicle safety performance," Report TP 13678-E, Transport Canada, 2000.

[2] C. Kwon, "Conditional value-at-risk model for hazardous materials transportation," in Proceedings of the Winter Simulation Conference (WSC '11), pp. 1703-1709, December 2011.

[3] T. T. Treichel, J. P. Hughes, C. P. L. Barkan et al., "Safety performance of tank cars in accidents: probabilities of lading loss," RSI-AAR Railroad Tank Car Safety Research and Test Project, Association of American Railroads, Washington, DC, USA, 2006.

[4] M. I. Salem, H. Victor, M. Mucino et al., "Review of parameters affecting stability of partially filled heavy-duty tankers," SAE Technical Paper 1999-01-3709, 1999.

[5] X. Kang, S. Rakheja, and I. Stiharu, "Cargo load shift and its influence on tank vehicle dynamics under braking and turning," Heavy Vehicle Systems, vol. 9, no. 3, pp. 173-203, 2002.

[6] W. H. Wang, Q. Cao, K. Ikeuchi, and H. Bubb, "Reliability and safety analysis methodology for identification of drivers' erroneous actions," International Journal of Automotive Technology, vol. 11, no. 6, pp. 873-881, 2010.

[7] W. Wang, W. Zhang, H. Guo, H. Bubb, and K. Ikeuchi, "A safetybased approaching behavioural model with various driving characteristics," Transportation Research C, vol. 19, no. 6, pp. 1202-1214, 2011.

[8] F. Solaas and O. M. Faltinsen, "Combined numerical and analytical solution for sloshing in two-dimensional tanks of general shape," Journal of Ship Research, vol. 41, no. 2, pp. 118129, 1997.

[9] K. Modaressi-Tehrani, S. Rakheja, and I. Stiharu, "Threedimensional analysis of transient slosh within a partly-filled tank equipped with baffles," Vehicle System Dynamics, vol. 45, no. 6, pp. 525-548, 2007.

[10] L. Strandberg, "Lateral stability of road tankers," VIT Report 138A, National Road and Traffic Research Institute, Linköping, Sweden, 1978.

[11] R. Ranganathan, S. Rakheja, and S. Sankar, "Steady turning stability of partially filled tank vehicles with arbitrary tank geometry," Journal of Dynamic Systems, Measurement and Control, vol. 111, no. 3, pp. 481-489, 1989.

[12] R. Ranganathan, "Rollover threshold of partially filled tank vehicles with arbitrary tank geometry," Proceedings of the Institution of Mechanical Engineers D, vol. 207, no. 3, pp. 241244, 1993.

[13] S. Sankar and S. Surial, "Sensitivity analysis approach for fast estimation of rollover stability of heavy articulated vehicles during steady state turning," Heavy Vehicle Systems, vol. 1, no. 3, pp. 282-303, 1994.

[14] C. B. Winkle, "Rollover of heavy commercial vehicles," Report UMTRI-99019, 2001.

[15] X. Li -S, X. Zheng -L, and H. Liu -F, "Improved algorithm on roll stability evaluation of partially filled tractor-tank semitrailer," Journal of Jilin University, vol. 42, no. 5, pp. 1089-1094, 2012 (Chinese).

[16] K. Modaressi-Tehrani, S. Rakheja, and R. Sedaghati, "Analysis of the overturning moment caused by transient liquid slosh inside a partly filled moving tank," Proceedings of the Institution of Mechanical Engineers D, vol. 220, no. 3, pp. 289-301, 2006.

[17] X. L. Zheng, X. S. Li, and Y. Y. Ren, "Analysis of rollover threshold for partially-filled tank vehicles impacted by transient liquid 
sloshing," Journal of Beijing Institute of Technology, vol. 21, supplement 2, pp. 169-174, 2012.

[18] F. T. Dodge, "Analytical representation of lateral sloshing by equivalent mechanical models," in The Dynamic Behavior of Liquids in Moving Containers, H. N. Abramson and S. Silverman, Eds., chapter 6, National Aeronautics and Space Administrator, Washington, DC, USA, 1966.

[19] H. N. Abramson, W. H. Chu, and D. D. Kana, "Some studies of nonlinear lateral sloshing in rigid containers," Journal of Applied Mechanics, vol. 33, pp. 777-784, 1966.

[20] M. I. Salem, Rollover Stability of Partially Filled Heavy-Duty Elliptical Tankers Using Trammel Pendulums to Simulate Fluid Sloshing [Ph.D. thesis], West Virginia University, Department of Mechanical and Aerospace Engineering, 2000.

[21] X.-L. Zheng, X.-S. Li, and Y.-Y. Ren, "Equivalent mechanical model for lateral liquid sloshing in partially filled tank vehicles," Mathematical Problems in Engineering, vol. 2012, Article ID 162825, 22 pages, 2012.

[22] L. Dai, L. Xu, and B. Setiawan, "A new non-linear approach to analysing the dynamic behaviour of tank vehicles subjected to liquid sloshing," Proceedings of the Institution of Mechanical Engineers K, vol. 219, no. 1, pp. 75-86, 2005. 


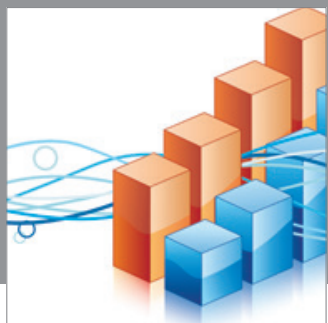

Advances in

Operations Research

mansans

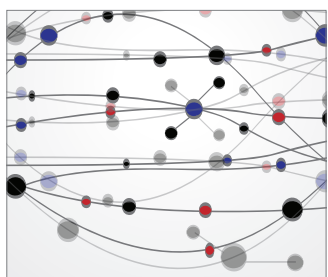

The Scientific World Journal
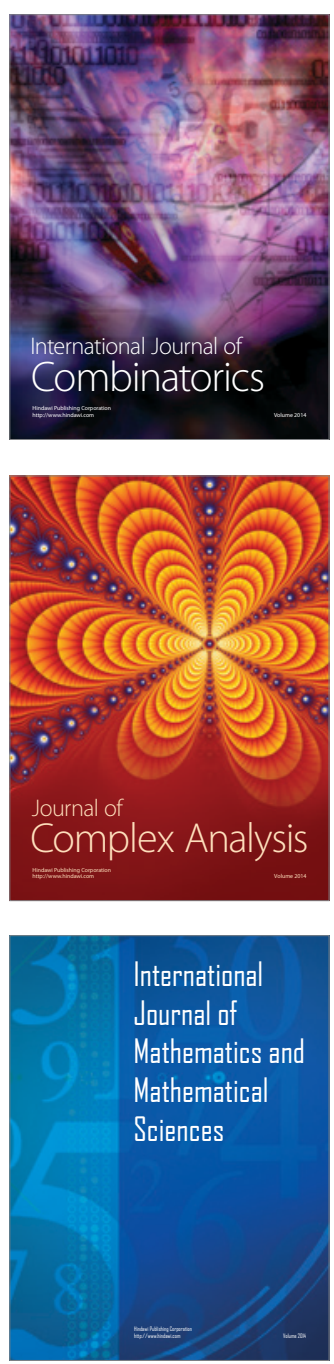
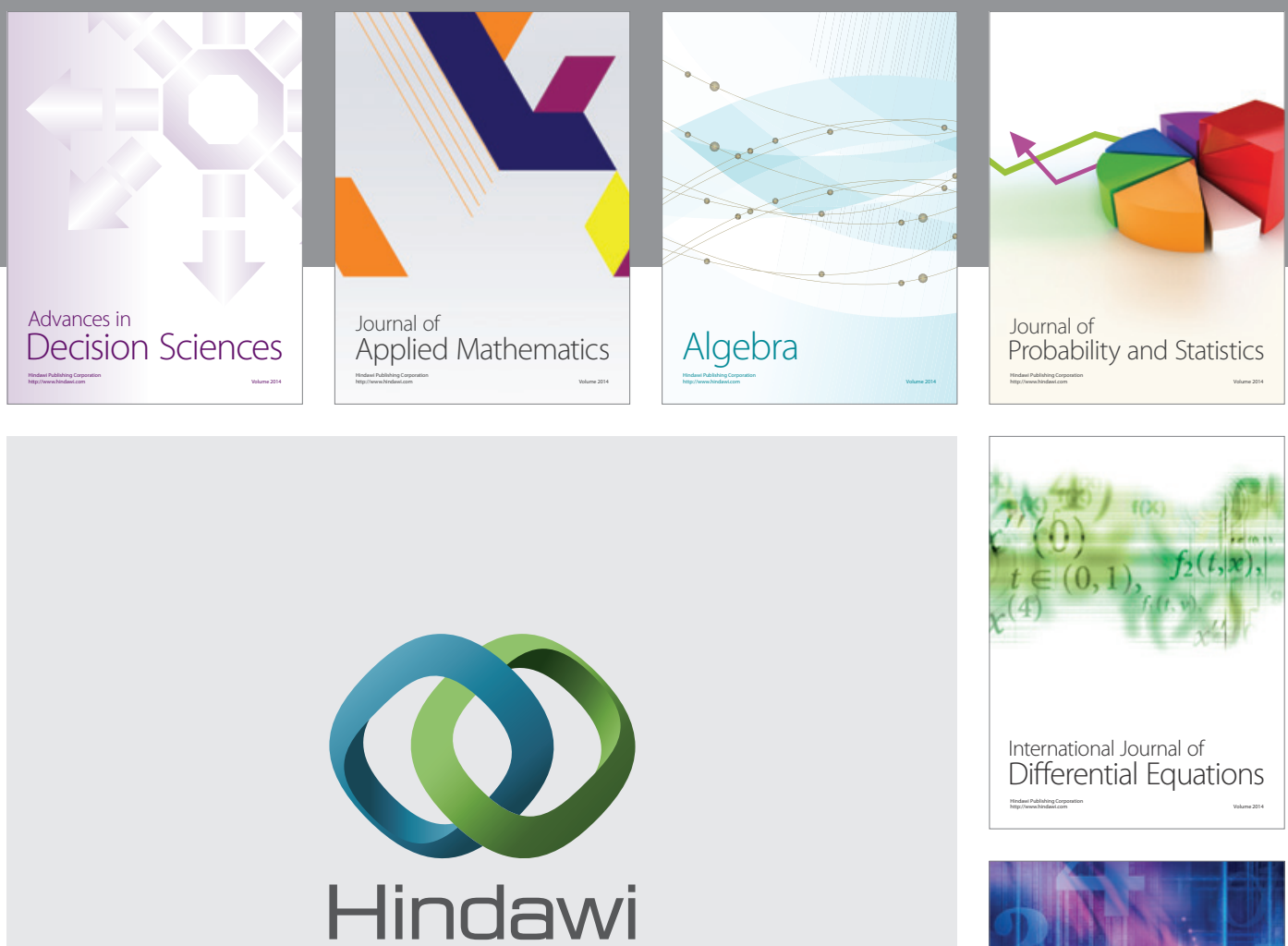

Submit your manuscripts at http://www.hindawi.com
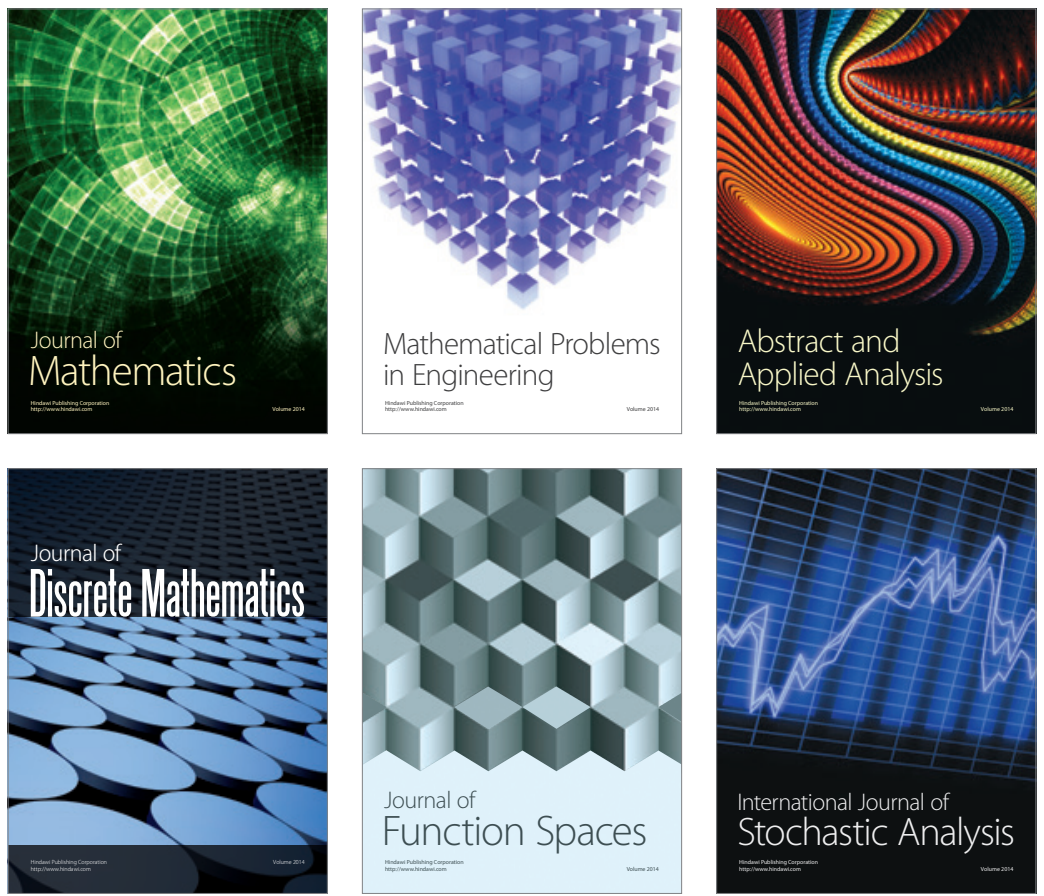

Journal of

Function Spaces

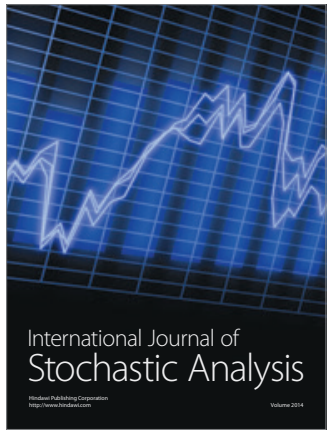

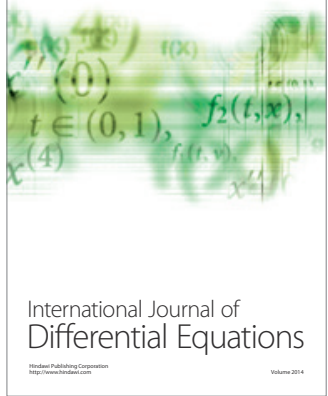
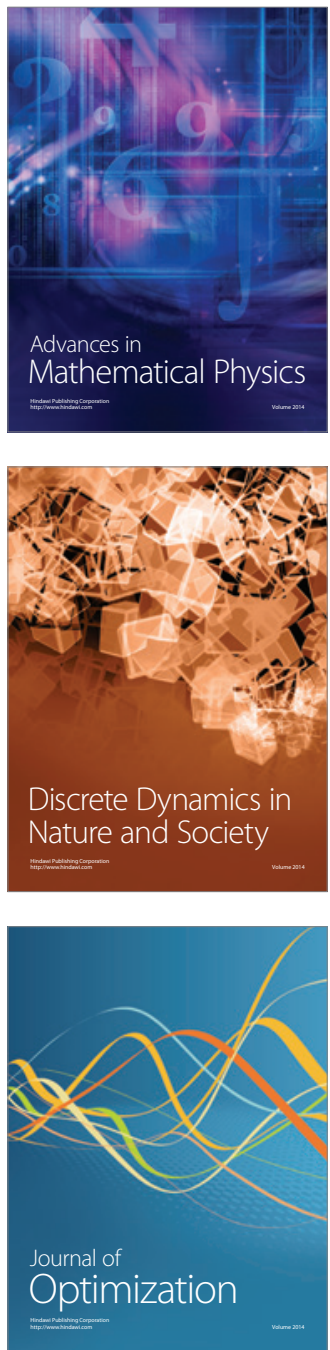